Article

\title{
Experimental and Numerical Study of the Aerodynamic Characteristics of an Archimedes Spiral Wind Turbine Blade
}

\author{
Kyung Chun Kim ${ }^{1}$, , Ho Seong Ji ${ }^{1}$, Yoon Kee Kim ${ }^{1}$, Qian Lu ${ }^{1}$, Joon Ho Baek ${ }^{2}$ and \\ Rinus Mieremet ${ }^{3}$
}

1 School of Mechanical Engineering, Pusan National University, Jangjeon-dong, Geumjeong-Gu, Busan 609-735, Korea; E-Mails: hsji@pusan.ac.kr (H.S.J.); yygkim@pusan.ac.kr (Y.K.K.); lqcygosla@pusan.ac.kr (Q.L.)

2 Department of Engineering Research, Eastern Star Cooperation Real Time Services (ESCO RTS), Doosanventuredigm, B112, Pyeongchon-Dong, 126-1, Dongan-Gu, Anyang, Gyeonggi-Do 431-070, Korea; E-Mail: neo100po@empas.com

3 The Archimedes, Looiershof 30, Rotterdam 3024 CZ, The Netherlands;

E-Mail: mieremetsolutions@yahoo.com

* Author to whom correspondence should be addressed; E-Mail: kckim@ pusan.ac.kr; Tel.: +82-51-510-2324; Fax: +82-51-515-7866.

External Editor: Frede Blaabjerg

Received: 9 May2014; in revised form: 29 October 2014 / Accepted: 14 November 2014 / Published: 26 November 2014

\begin{abstract}
A new type of horizontal axis wind turbine adopting the Archimedes spiral blade is introduced for urban-use. Based on the angular momentum conservation law, the design formula for the blade was derived using a variety of shape factors. The aerodynamic characteristics and performance of the designed Archimedes wind turbine were examined using computational fluid dynamics (CFD) simulations. The CFD simulations showed that the new type of wind turbine produced a power coefficient $\left(C_{p}\right)$ of approximately 0.25 , which is relatively high compared to other types of urban-usage wind turbines. To validate the CFD results, experimental studies were carried out using a scaled-down model. The instantaneous velocity fields were measured using the two-dimensional particle image velocimetry (PIV) method in the near field of the blade. The PIV measurements revealed the presence of dominant vortical structures downstream the hub and near the blade tip. The interaction between the wake flow at the rotor downstream and the induced velocity due to the tip vortices were strongly affected by the wind speed and resulting rotational speed of the blade.
\end{abstract}


The mean velocity profiles were compared with those predicted by the steady state and unsteady state CFD simulations. The unsteady CFD simulation agreed better with those of the PIV experiments than the steady state CFD simulations.

Keywords: Archimedes spiral wind blade; particle image velocimetry; tip vortex; wake; computational fluid dynamics (CFD); unsteady analysis

\section{Introduction}

To secure energy supply issues and address climate change, reductions of Greenhouse Gas (GHG) emissions, biodiversity protection, development of renewable technologies, energy conservation, and efficiency improvements are becoming increasingly important. Among the renewable resources, wind energy is a relatively mature technology with enormous potential for commercialization and mass production. The major application of wind power is electricity generation from large grid-connected wind farms [1]. With the expansion of the power grid and the reduction of electricity scarce areas, small-scale wind turbines are now being applied in several countries and in many fields, such as city road lighting, mobile communication base stations, offshore aquaculture, and sea water desalination [2].

Small scale wind turbines installed within the built environment are classified as micro generation technology. Some studies into urban energy generation have been performed in the United Kingdom. One study reported that it is possible to predict with a high degree of accuracy the expected financial payback period for a typical domestic household [3]. Small scale wind turbines can be classified as horizontal axis wind turbines (HAWT) and vertical axis wind turbines (VAWT). Ahmed studied the feasibility of small scale wind turbines including low noise, low cost, safe and easy operation for power generation by Computational Fluid Dynamics (CFD) and a wind tunnel test [4]. Small scale wind turbines with less than $10 \mathrm{~kW}$ of installed power were analyzed by comparing the power curves [4]. A variety of wind turbines were analyzed [5-8]. These studies reported the effects of the blade geometry on the power curve, the turbine's rated power related to its swept area, the total electricity production, and the pay-back period. Arens and Williams conducted numerical analysis on Darrieus VAWTs to characterize the flow field in the rooftop area of buildings with different shapes and geometrical proportions [9]. In their study, the feasibility of the rooftop installation of Darrieus VAWTs was evaluated and the analyses were combined in an energy-oriented study [10,11]. The effects of the pitch angle and blade camber on the flow characteristics and performance of a small-size Darrieus VAWT system driven at a uniform wind speed $(10 \mathrm{~m} / \mathrm{s})$ were investigated using a simulation model [12]. Scheurich and Brown [13] investigated numerically the aerodynamics of VAWTs in unsteady wind conditions using the vorticity transport model. The inherent unsteadiness in the blade aerodynamic loading because of the continuous variation of the angle of attack on the blades is shown to be larger than that are induced by the unsteadiness in the wind conditions. Edwards et al. [14] reported 2D PIV measurements and 2D CFD simulation of the performance and flow physics and of a small-scale VAWT. They found that the most significant CFD-PIV differences are observed in predicting flow reattachment.

The Archimedes spiral wind turbine, which is a new HAWT concept, was designed using the Archimedes spiral principles. Unlike traditional HAWTs, which use the lift force to take power from 
wind energy, the Archimedes spiral wind turbine uses both the lift and drag force. The Archimedes spiral wind turbine can utilize the kinetic energy from wind energy. This special structure determines the special aerodynamic characteristics of small scale wind turbines. In particular, the advantages of the Archimedes spiral structure will be more obvious in many circumstances, such as around buildings, because the wind turbine operates at low wind speeds. The wind direction in an urban environment changes constantly but the Archimedes wind turbine follows the wind direction automatically because the yaw is passively controlled due to the drag force. Other advantages include low noise because of the relatively low rotational speed. The disadvantage of the Archimedes wind turbine is the high thrust force compared to a propeller-type conventional HAWT. In 2009, Timmer and Toet carried out fundamental research to examine the potential and optimal power output of the Archimedes spiral small wind turbine [15]. The highest efficiency measured in their study was $12 \%$. Lu et al. [16] recently developed a design method for the Archimedes spiral wind turbine blade and performed a numerical simulation using ANSYS CFX v12.1. To the best of the authors' knowledge, there are no reports on the aerodynamic characteristics of Archimedes spiral wind turbines.

In this study, a theoretical approach was introduced to design a small scale Archimedes wind turbine based on the angular momentum conservation law and the performance of the designed Archimedes wind turbine was evaluated using the CFD method. Particle image velocimetry (PIV) was used to examine the aerodynamic characteristics of the Archimedes spiral wind turbine blade, validate the CFD method as a design tool of the Archimedes wind turbine, and understand the fluid dynamics in the near wake. The aerodynamic characteristics of the Archimedes spiral wind turbine, such as the ensemble averaged velocity field at different wind speeds, turbulence kinetic energy distribution in the tip vortex of the wind turbine, and instantaneous velocity fields, were obtained from an experimental study and compared with those calculated using the CFD method.

\section{Design of the Archimedes Spiral Wind Turbine}

Figure 1 shows a schematic diagram of a $0.5 \mathrm{~kW}$ class Archimedes spiral wind turbine. The three blades were connected to each other with $120^{\circ}$ and each blade has a symmetric arrangement around the shaft and its shape was similar to a triangular pyramid. The outer diameter of the $0.5 \mathrm{~kW}$ class Archimedes wind turbine was $1.5 \mathrm{~m}$. The aerodynamic equation for torque prediction with regard to the angular velocity was estimated using the following four assumptions:

1) Incompressible and steady flow;

2) The fluid moves to the control volume parallel to the rotating axis;

3) The quantities of mass flow at the three outlet boundaries were the same as each other;

4) Relative outlet velocity was constant in outlet boundary and the direction of the velocity was parallel to the tangential direction at the blade edge.

To provide the torque and power of the small wind turbine with a spiral wind blade, the momentum equation for the control volume employed in this study can be defined using Equation (1):

$$
\frac{\partial}{\partial t} \int_{C V}(r \times V) \rho d \forall+\int_{C S}(r \times V) \rho V \cdot \hat{n} d A=\sum(r \times F)
$$


Equation (1) is the angular momentum equation based on the control volume. From the steady state assumption, the first term in Equation (1) becomes zero because of the time independence, and Equation (1) can be simplified as Equation (2):

$$
\int_{C V}(r \times V) \rho d V \cdot \hat{n} d A=\sum(r \times F)
$$

The continuity equation on a fixed control volume is represented by Equation (3):

$$
\frac{\partial}{\partial t} \int_{C V} \rho d V+\int_{C V} \rho W \cdot \hat{n} d A=0
$$

From the steady state assumption, the first term in Equation (3) becomes zero because of the constant density. In addition, the second term means the sum of the quantities of mass flow that pass through the control volume. Equation (3) can be simplified to Equation (4):

$$
-\dot{m}_{\text {in }}+\dot{m}_{\text {out }}=0
$$

Figure 1. Schematic diagram of the Archimedes spiral wind turbine blade: (a) Side-view of the Archimedes spiral wind turbine blade; (b) Geometry of a $0.5 \mathrm{~kW}$ Archimedes wind turbine blade.

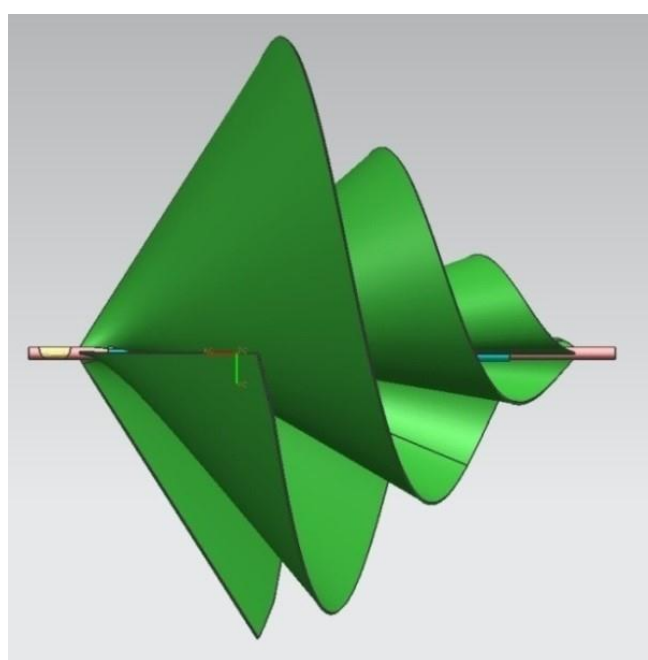

(a)

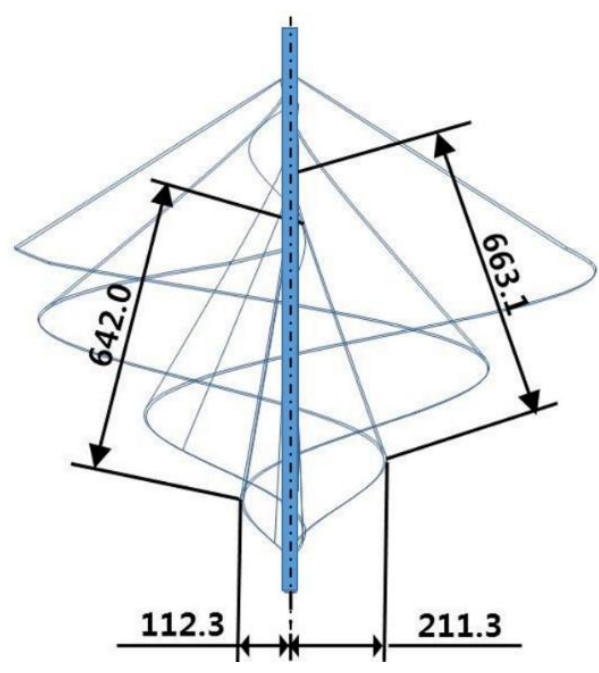

(b)

The shape parameters can be defined for the equation development. The shape parameters include $\gamma$, $R_{1}$, and $S_{1}$, as shown in Figure 2. First, $\gamma$ is defined by the angle between the rotational axis and the tip of the blade, $\mathrm{R}_{1}$ means the vertical distance of the outer blade tip from the rotational axis, $\mathrm{R}_{2}$ means the vertical distance of the inner blade tip from the rotational axis, and $\mathrm{S}_{1}$ means the horizontal distance between the front blade tip and the root of the spiral blade. The mass flow at flow in and flow out is defined by Equations (5) and (6) using pre-defined shape parameters:

$$
\begin{gathered}
\dot{m}_{\text {in }}=\rho U_{\infty} \sin \gamma \times \pi\left(\frac{R_{1}+R_{2}}{2}\right) S_{1} \\
\dot{m}_{\text {out }}=3 \rho A_{\text {out }} W_{\theta}
\end{gathered}
$$

where $W_{\theta}$ means the tangential component for the relative velocity. Additional shape parameters are derived in detail in the Appendix. 
Finally, the torque of the blade can be expressed using Equation (7):

$$
\therefore T_{\text {shaft }}=3 \rho V_{\theta}^{2} \times \frac{\left(R_{2}-R_{1}\right)}{6 L_{1}\left(L_{2}-L_{1}\right)} \times\left[R_{2}\left(2 L_{2}^{3}+L_{1}^{3}+L_{1} L_{2}^{3}\right)-L_{1} R_{1}\left(L_{1}+L_{2}\right)^{2}\right]
$$

The power is a multiplication of the torque and angular velocity, and can be expressed using Equation (8):

$$
\text { Power }=T_{\text {shaft }} \omega
$$

Figure 2. Shape parameter of the Archimedes spiral wind turbine blade.

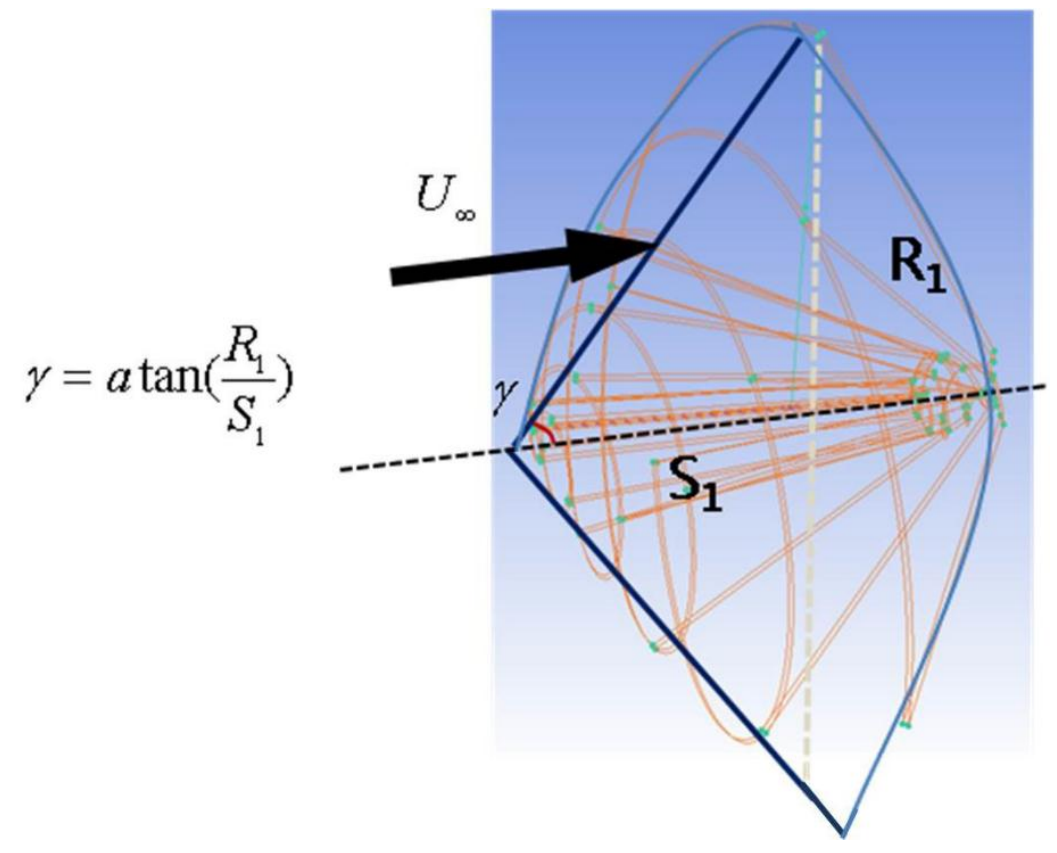

\section{Numerical and Experimental Methods}

\subsection{Numerical Method}

CFD analysis was conducted using a commercial multi-purpose CFD solver, ANSYS CFX. The code is idealized according to the Reynolds Averaged Navier-Stokes Equation and the Finite Volume Method of governing equations. The k- $\omega$-Shear Stress Transport (SST) turbulence model was used to predict the separation of flow accurately [17].

Figure 3 presents the mesh generation including the rotating domain and stationary domain. CFX separates the grid into the entire domain of using the Multiple Frames of Reference (MFR) method, rotating domain of the spiral blade and stationary domain of the imaginary wind tunnel simulation. Two different numerical simulations were carried out. One was to predict the performance of the designed $0.5 \mathrm{~kW}$ Archimedes spiral wind turbine, and the other was to validate the CFD results for the 1/10 scaled down wind turbine model used for the PIV experiment. The total cell number for the $0.5 \mathrm{~kW}$ wind turbine was approximately $2,300,000$ and 1,000,000 for the stationary domain, and 1,300,000 for the rotating domain. The cell number for the scaled down model was approximately 292,935 on the rotating domain, and approximately 50,106 on the stationary domain, giving a total of 343,041 cells. Grid tests were performed for both cases and the grid independency was confirmed. Very fine grids were used near the 
turbine blade to resolve the near wake structures. The $\mathrm{Y}^{+}$value of the blade was approximately 4 except for the root region of the blade.

Figure 3. Meshing of the rotating and stationary domain.

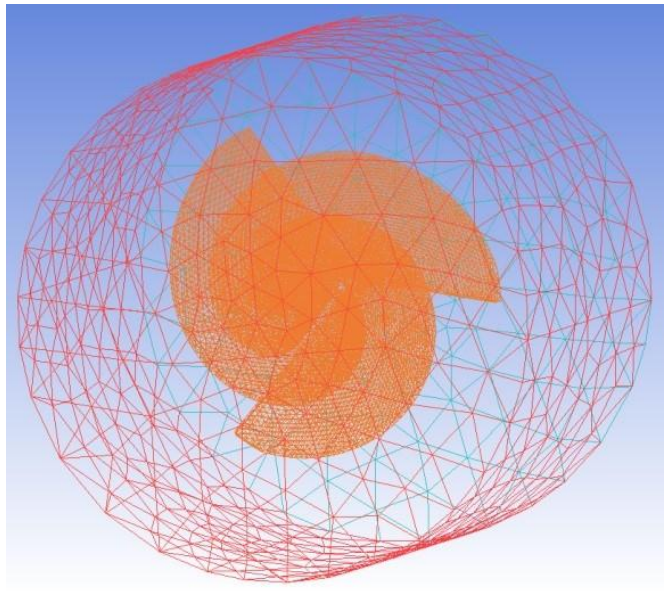

(a)

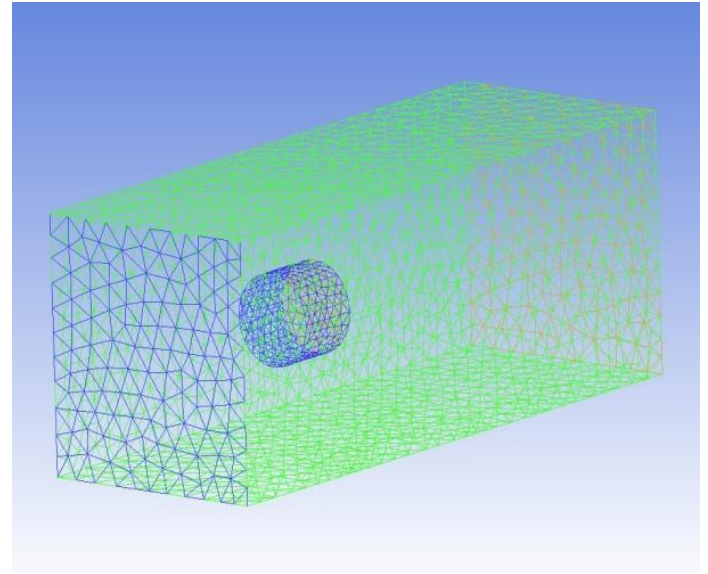

(b)

Figure 4 shows the grid constructed by the ICEM CFD and established on CFD-Pre with the boundary conditions. To reduce the computation time, CFD was conducted with an imaginary wind tunnel. The size of the imaginary wind tunnel was $0.4 \mathrm{~m} \times 0.4 \mathrm{~m} \times 1.5 \mathrm{~m}$. The diameter of the Archimedes wind turbine blade was $0.15 \mathrm{~m}$. The blockage ratio based on the swept frontal area was $11.0 \%$. On the other hand, the area of the wind turbine when stationary is very small. If a blade thickness of $3 \mathrm{~mm}$ is considered, the blockage ratio is approximately $0.8 \%$.

Figure 4. Boundary condition setup for CFX-Pre.

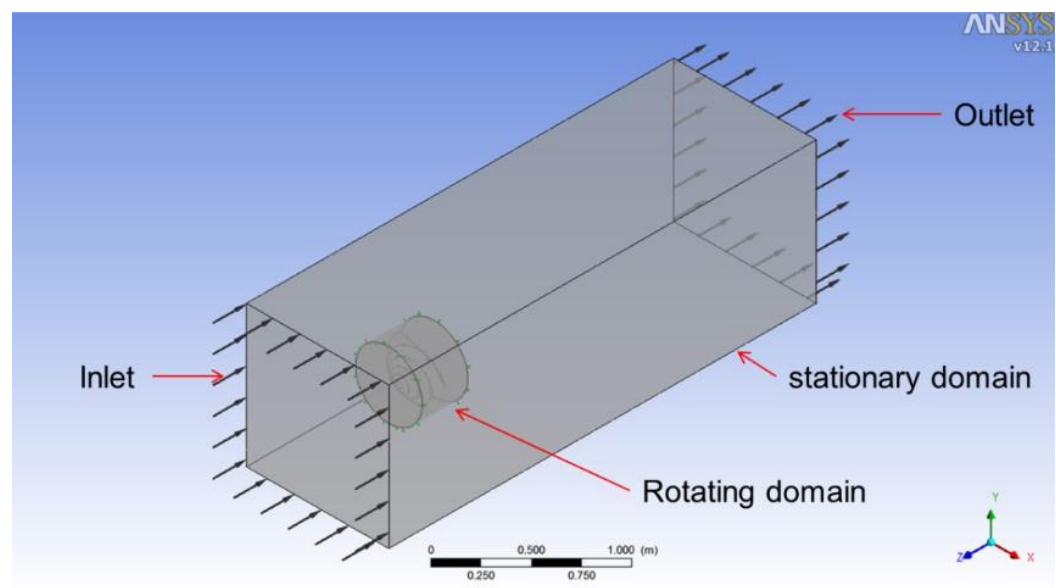

The literature suggests that the blockage ratio based on the swept frontal area is less than $14 \%$, the effect of the blockage ratio is negligible [18]. In this simulation, three cases were chosen to predict the aerodynamic characteristics with inlet wind velocities of 3.5, 4 and $4.5 \mathrm{~m} / \mathrm{s}$, and rotating velocities of 300, 400 and $500 \mathrm{rpm}$, respectively. The outlet boundary was set as the pressure outlet of $0 \mathrm{~Pa}$ (gauge pressure). Free-slip conditions were applied to the top, bottom, and side surfaces. This means that there is no wind speed gradient normally and no flow across the top, bottom, and side surfaces. The conservative interface flux for mass and momentum was applied to three sharing surfaces of the rotating 
and stationary domain, and the inlet and outlet air flow through the boundary surface automatically was calculated. The General Grid Interface (GGI) interface boundary condition was used.

\subsection{Experimental Method}

The aerodynamic characteristics and evolution of the tip vortex structures in the near wake of the Archimedes spiral wind turbine were examined by quantitative flow visualization experiments using the conventional two-dimensional PIV technique. Figure 5 presents a schematic diagram of the experimental setup and the PIV field of view used in this study. The experimental apparatus consisted of an Archimedes spiral wind blade model, $150 \mathrm{~mm}$ in diameter, an open type wind tunnel, a mini Nd:YAG laser (U wave) as the light source of PIV measurement, a digital 12 bit CCD camera (PCO sensicam QE) with a $60 \mathrm{~mm}$ lens for particle image acquisition, a Laskin nozzle for olive oil aerosol generation, and a remote optical sensor with a display.

Figure 5. Experimental setup for the PIV measurement and Field of View.

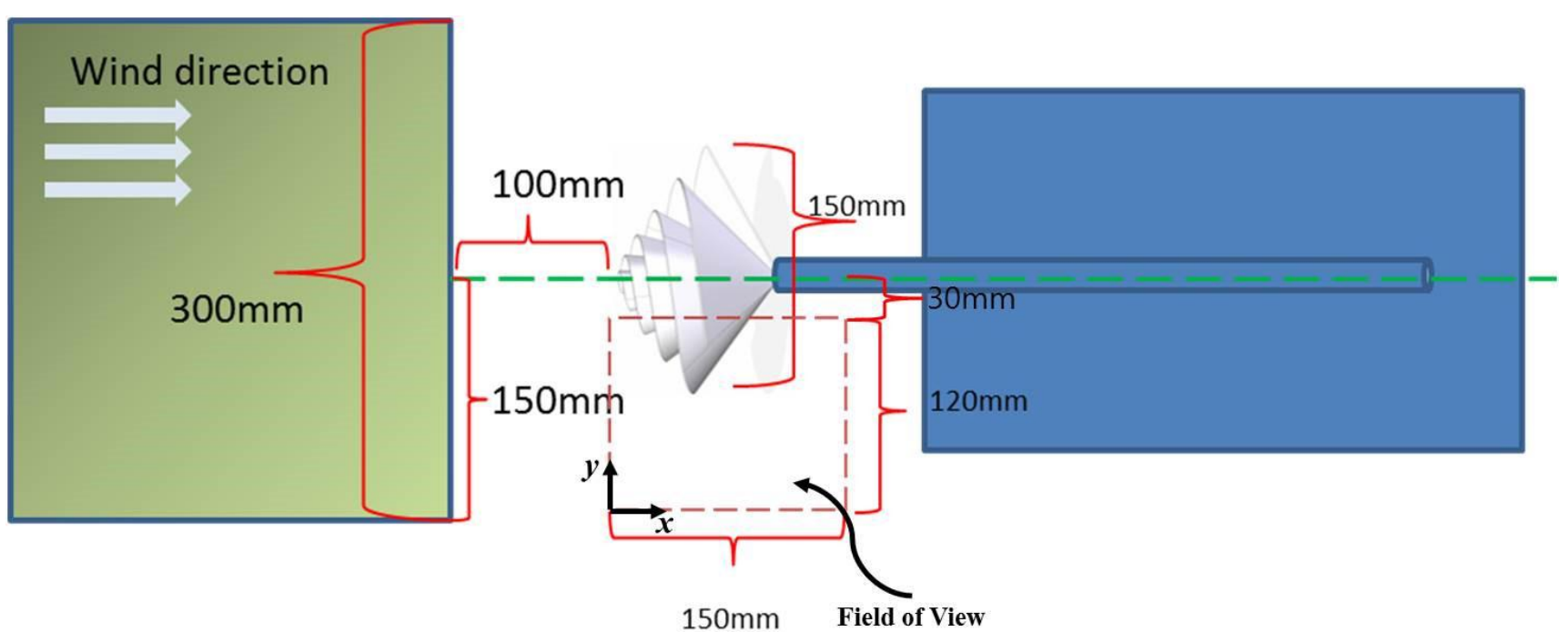

The laser beam, which was generated from the $U$ wave mini-YAG laser, was transformed to a light sheet (1 mm thickness) using cylindrical and spherical lenses and illuminated on the observation plane in a manner of side scattering. The laser power was $150 \mathrm{~mJ}$ per pulse. The observation field of view was $150 \times 120 \mathrm{~mm}^{2}$, which corresponds to $1280 \times 1024$ pixels. The location of the field of view for the PIV measurement is shown in Figure 5. A digital 12 bit CCD camera (PCO sensicam QE camera) fitted with a $60 \mathrm{~mm}$ lens was setup vertically under the Archimedes wind turbine to capture the particle images in the $\mathrm{x}-\mathrm{y}$ plane. The exposure time was controlled appropriately according to the wind speed. Olive oil droplets whose diameter was $2 \mu \mathrm{m}$ used for the PIV tracer particles were then generated by a Laskin nozzle and provided at the upstream areas of the wind tunnel.

The experimental model of the Archimedes spiral wind turbine was a 1:10 scale model of a $0.5 \mathrm{~kW}$ class wind turbine. To prevent deformation under the flow conditions, the experimental blade model was $3 \mathrm{~mm}$ in thickness and was manufactured using a rapid prototype (RP) 3D printer with a suitable resin-based polymer. According to the specifications of the 3D printer, the surface roughness is less than $0.25 \mathrm{~mm}$. The experimental model was placed $100 \mathrm{~mm}$ downstream from the exit of the wind tunnel contraction nozzle. The tip point of the experimental model was placed in the center line of the wind 
tunnel and was maintained during the entire experiment. The area of the outlet cross section of the open type wind tunnel was $300 \times 300 \mathrm{~mm}^{2}$, which is two times larger than the diameter of the experimental wind turbine model. In this experiment, the wind turbine model was placed between 0.33 and 0.73 times of nozzle hydraulic diameter from the exit, which is within the acceptable region for an open-jet wind tunnel test [19]. When the wind turbine model is rotating, the real solidity is about $1.8 \%$ of the nozzle area. We confirmed that there was no severe deflection of outer streamlines by the PIV measurement. The mean velocity profile and turbulence intensity was measured at the exit of the open circuit wind tunnel using the same PIV system. The mean velocity was uniform (less than $1 \%$ standard deviation) except for the edge of the exit. On the other hand, the wind model was located in the uniform flow region (in the potential core). The measured turbulence level was $2 \%$.

The instantaneous velocity fields were calculated from 2000 particle images acquired for the observation window under each experimental condition. The instantaneous flow fields were obtained to examine mean velocity fields, turbulent kinetic energy distribution and the signature of the tip vortex. The interrogation window size was $32 \times 32$ pixels, the window overlap was $50 \%$, and a 2 frame cross-correlation method was used. Through the post processing procedure, the spurious vectors in the raw vector fields were removed using the magnitude difference technique with an $80 \%$ threshold and were then interpolated with a Gaussian convolution [20].

An optical tachometer was used to measure the rotational speed of the wind turbine model at the end of the main shaft. The wind turbine model was in free run mode because there was no load except for bearing friction, which was negligible. To measure the rotational speed and capture the particle images in the cases of different wind directions, the position of the experiment was adjusted based on the front tip point of the wind turbine model.

\section{Results and Discussion}

\subsection{Results from CFD Analysis}

There are several methods to predict the aerodynamic characteristics of wind turbines [21,22]. In this study, the full 3D CFD approach with the rotating blade was adopted using the commercial CFD software, ANSYS-CFX.

Figure 6 shows the $C_{p}$ versus tip speed ratio curve obtained by the CFD analysis when the velocity was changed from 5 to $12 \mathrm{~m} / \mathrm{s}$ for the $0.5 \mathrm{~kW}$ Archimedes wind turbine. The in-flow wind profile was assumed to be uniform. The maximum power coefficient observed near a tip speed ratio of approximately 2.5 was approximately 0.25 . The power coefficient curves showed a similar tendency regardless of the velocities. The modern urban-usage 3-blade and Darrieus blade type wind turbines have high rotor efficiency up to 0.25 in the high tip speed ratio range $[8,10]$. This means that high efficiency can be achieved in the case of a high wind speed. On the other hand, in an urban environment, the wind velocities are usually less than $3 \mathrm{~m} / \mathrm{s}$ [8]. However, the local wind speed can vary substantially depending on the location, for example it may be much higher above the roof of a large building. The spiral wind blade with an Archimedes shape shows relatively high rotor efficiency compared to the aerodynamic performance of the other blades like the Savonius type rotor in the lower tip speed ratio range [23]. In addition, the Archimedes spiral blade had high $C_{p}$ values over a wider range of tip speed ratios. 
Figure 6. $\mathrm{C}_{\mathrm{p}}$-Tip speed ratio with respect to the wind velocity.

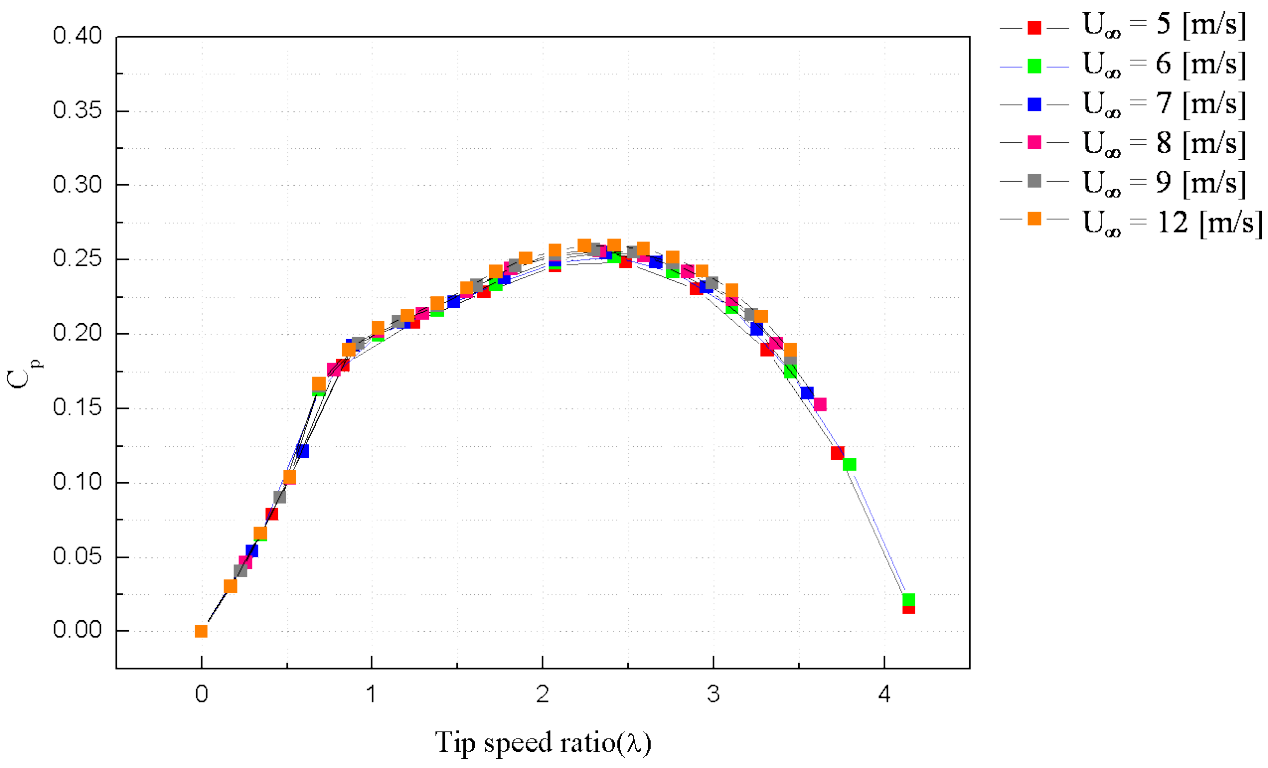

Figure 7 shows torque according to the rotating speed for a comparison of the theoretical prediction with the numerical results. In the case of the theoretical result, the torque for the rotating speed has a gradual decreasing tendency at $U=5 \mathrm{~m} / \mathrm{s}$. The results of the torque for wind power generation through numerical analysis showed a similar tendency to the theoretical analysis. From this result, the numerical analysis appears to show relatively good agreement at the operating rotational speed regions.

Figure 7. Comparison of the theoretical prediction with the numerical results $(U=5 \mathrm{~m} / \mathrm{s})$.

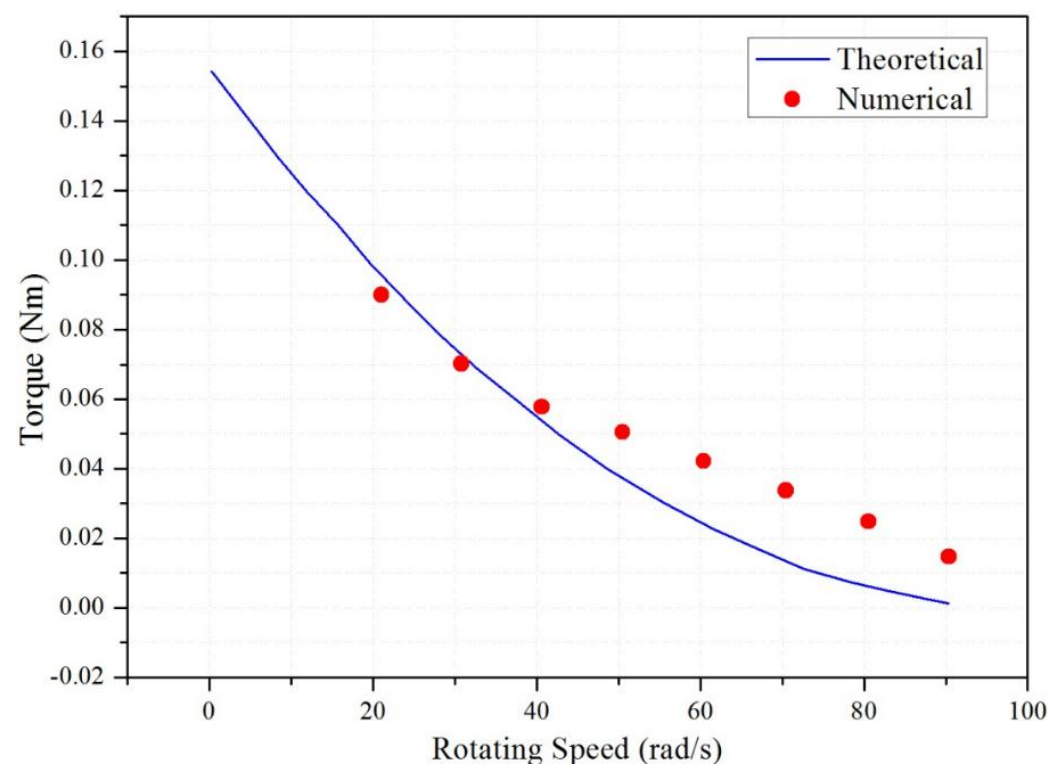

A comparative study of the CFD and PIV experiment was performed to validate the CFD results to be used as a design tool for the Archimedes wind turbine. For this purpose, the geometry of the wind turbine blade, the inlet velocity profile and rotating speeds of the blade for CFD analysis were the same as the experimental conditions.

Figure 8 shows the calculated ensemble averaged velocity fields of the overall flow field on the central plane of the Archimedes spiral wind turbine at three different wind speeds, which were characterized by 
the contours and velocity vectors. The rotating velocities measured at in-flow velocities of 3.5, 4.0 and $5.0 \mathrm{~m} / \mathrm{s}$ were 300,400 and $500 \mathrm{rpm}$, respectively. The corresponding tip speed ratios were $0.62,0.78$ and 0.79 , respectively. Remarkably, details of the flow behavior were obtained over a range of wind velocities. Indeed, the contours shown in these figures correspond to the turbulent wake shape.

Figure 8. Ensemble-averaged velocity fields obtained by the steady simulation: (a) $3.5 \mathrm{~m} / \mathrm{s}$ and $300 \mathrm{rpm}$; (b) $4 \mathrm{~m} / \mathrm{s}$ and $400 \mathrm{rpm}$; (c) $5 \mathrm{~m} / \mathrm{s}$ and $500 \mathrm{rpm}$.

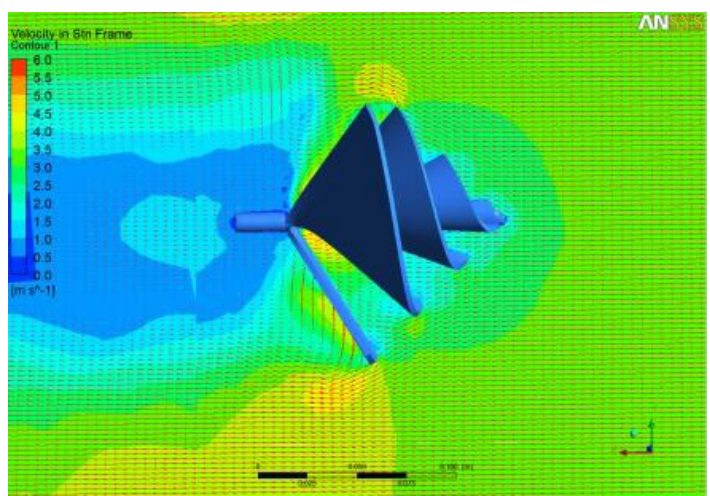

(a)

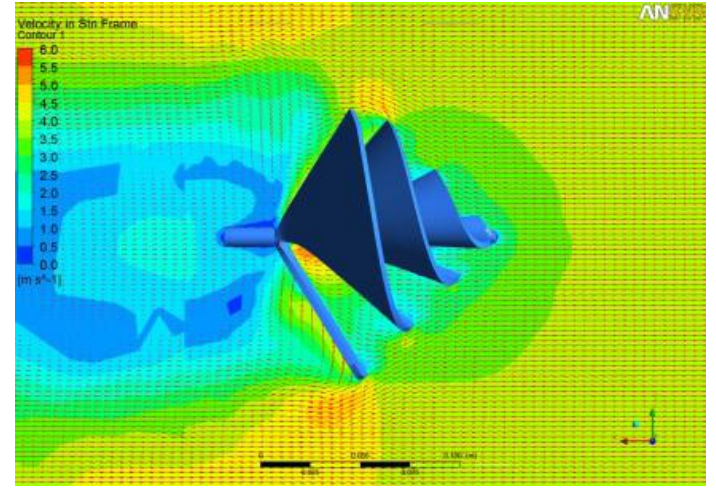

(b)

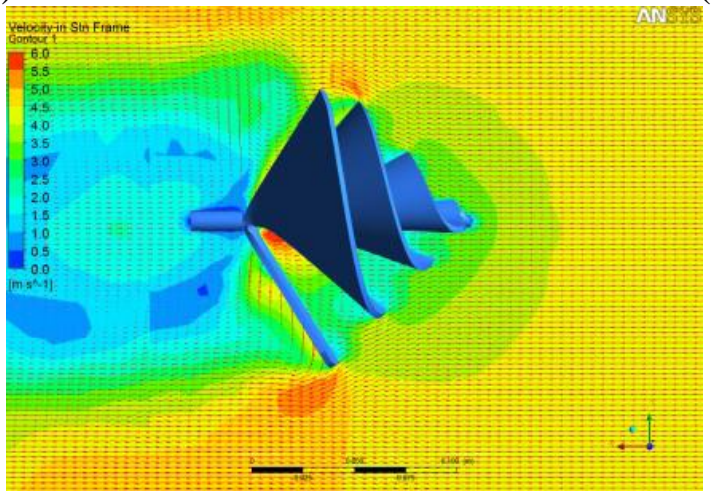

(c)

The highest velocities were observed in the vicinity of the rotor tip in the calculation domain. Because of the spiral effect, the velocities from the leading edge increased at the inner side of each blade. A recirculation zone with lower speeds was also observed in the wake regions because the incoming airflow was blocked by the hub cone and rotor. A circular accelerating zone existed behind the rotor, which resulted in a low pressure near the wall of the rotating domain. A low-speed region formed behind the hub of the rotor, indicating the wake region. The size of the recirculating zone was approximately one and half diameters long from the back side of the rotor and the maximum width of the wake region was equal to the rotor diameter.

Figure 9 shows the pressure distribution in the center plane of the wind turbine. The flow direction was from right to left. When the blade is rotating, there is a pressure difference between the pressure side and the suction side. Due to the spiral surface of the blades, the pressure difference (a force) generates torque. In general, the front side of the blade has higher pressure while the corresponding rear side has a lower pressure. 
Figure 9. Static pressure distribution obtained by the steady simulation (a) $3.5 \mathrm{~m} / \mathrm{s}$ and $300 \mathrm{rpm}$; (b) $4 \mathrm{~m} / \mathrm{s}$ and $400 \mathrm{rpm}$; (c) $5 \mathrm{~m} / \mathrm{s}$ and $500 \mathrm{rpm}$.

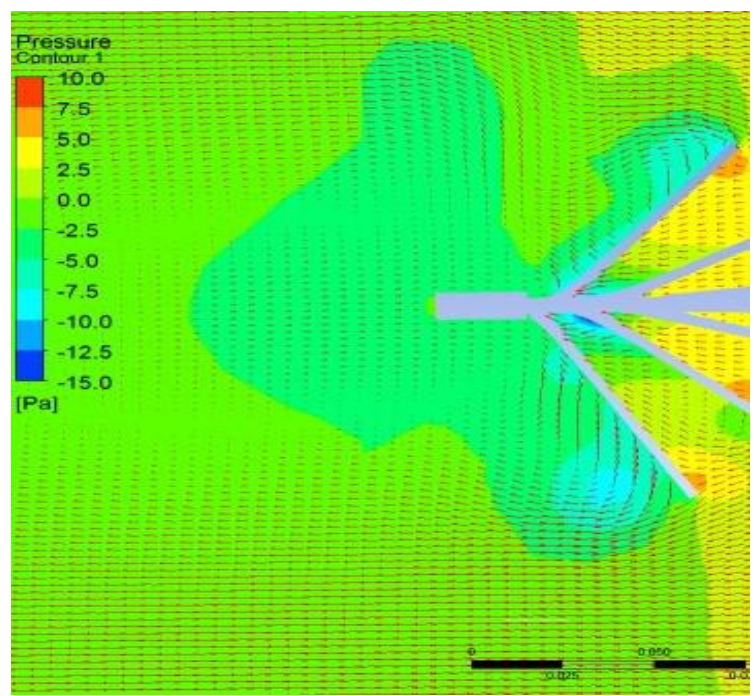

(a)

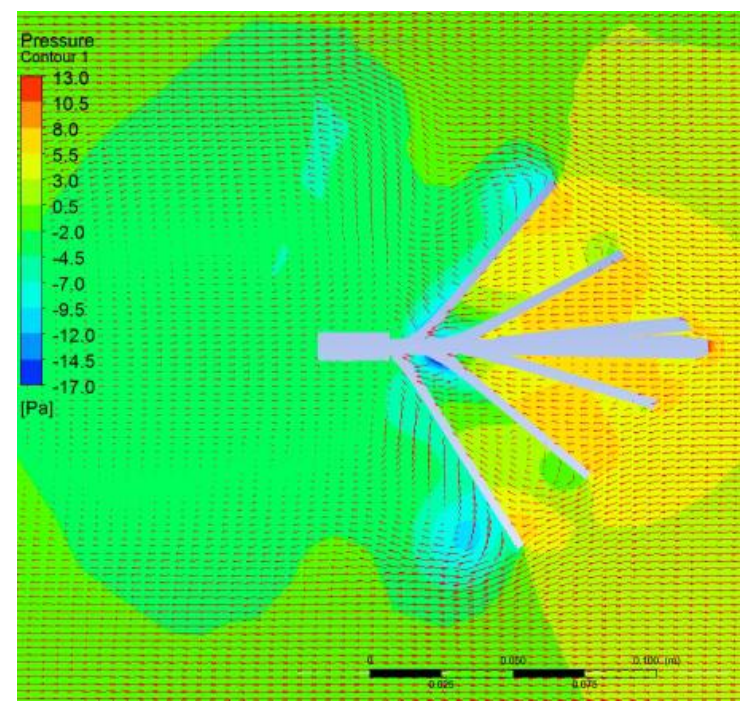

(b)

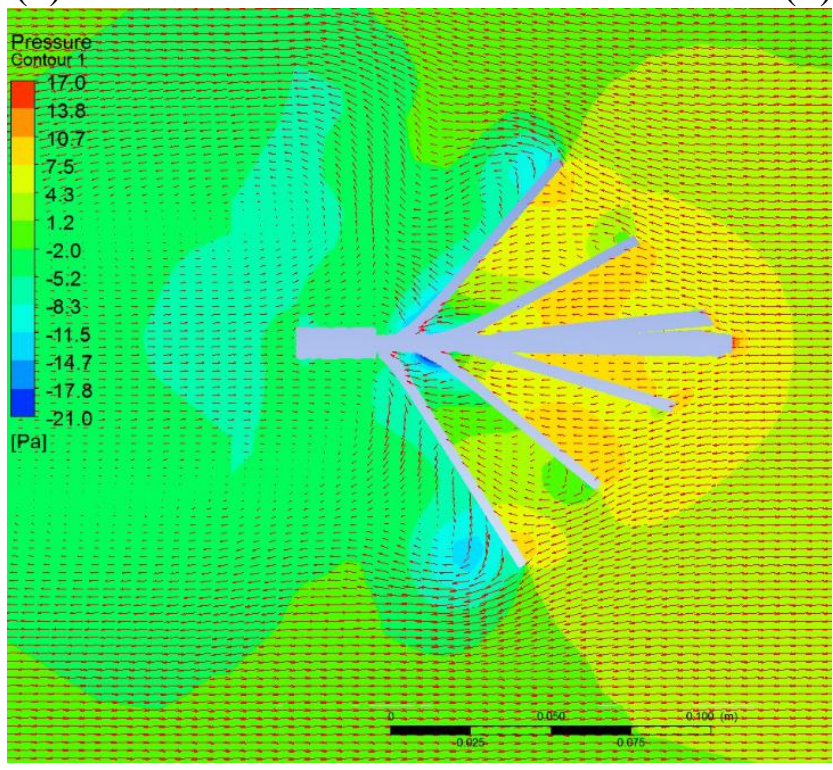

(c)

When the in-flow velocity increases, the pressure difference becomes larger. The pressure difference is large at the blade tip but small at the root region. This means that most of energy can be extracted near the blade tip like a three blade HAWT. The pressure differences between the root and tip were approximately $3 \mathrm{~Pa}$. On the suction side, however, the pressure differences were much higher. The pressure differences were more than $15 \mathrm{~Pa}$ (even more than $20 \mathrm{~Pa}$ at wind speeds of 4 and $5 \mathrm{~m} / \mathrm{s}$ ) lower at the tip than the root. Therefore, the pressure difference between the two sides at a section increase towards the tips of each blade. For the three cases, the rear side pressure is negative, so that thrust force can be exerted to the shaft.

Figure 10 shows the vortical structures represented by the isosurface of the swirl strength in case of different wind speeds. The swirl strength was calculated based on a three-dimensional unsteady simulation. The maximum swirl strength occurred in the tip vortex. A threshold swirl strength, which is $50 \%$ of the maximum strength, was selected and the vortical structures were rendered using iso-surface 
of the swirl strength. The near wake structure of the Archimedes spiral wind turbine was different from the structure of tip vortex generated by the general HAWT type wind turbine. Owing to the spiral shape of the blade, the evolution of the tip vortex developed in a spiral manner, and the core of the tip vortex was removed from the edge of the blade along the downstream direction. The swirl strength became stronger with increasing wind speed.

Figure 10. Vortical structures represented by the isosurface of the swirl strength (unsteady simulation, $5.0 \mathrm{~m} / \mathrm{s}$, and $500 \mathrm{rpm}$ ).

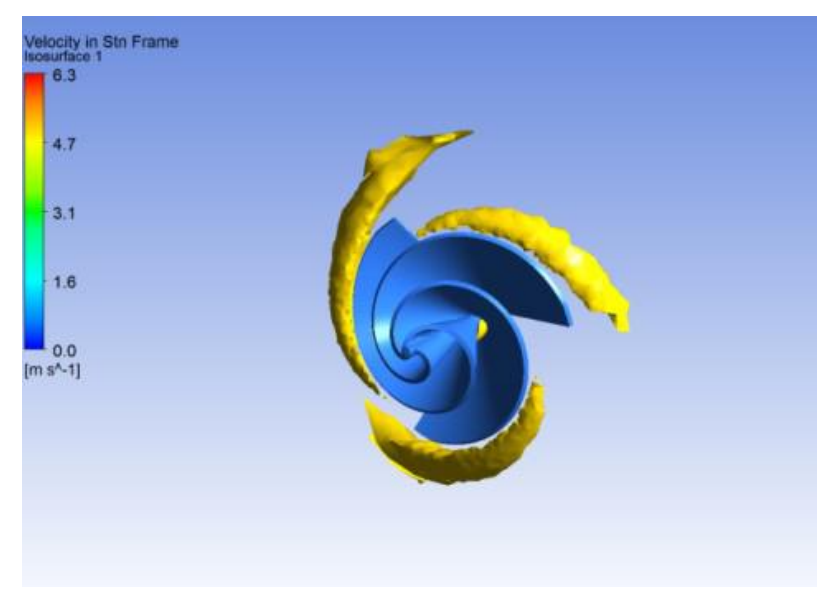

\subsection{Experimental Results}

Three speeds were measured at the exit of the open circuit wind tunnel, which are uniform in-flow velocities to the experimental model. Based on the diameter of the blade and uniform flow velocities, 3.5, 4.0 and $5.0 \mathrm{~m} / \mathrm{s}$, the Reynolds numbers were 34,770, 39,740 and 49,670, respectively.

Figure 11 shows the mean velocity contour obtained at a $0^{\circ}$ angle of attack. Owing to the presence of the Archimedes spiral blade, the decrease in mean velocity was observed while the incoming airflow passes through the surface of the blades. The streamwise momentum was converted to the angular momentum because of the rotation of the blade followed by a decrease in the mean velocity in this region. After passing through the blade, the air velocity was also decreasing. On the other hand, there was no flow separation because the flow passage through the blade was increasing. According to the momentum conservation law, the momentum deficit which is induced by the decreasing streamwise velocity after the wind turbine can be transformed to the power of the blade shaft. The maximum velocity region was located at the position of the maximum diameter of the blade, approximately one radius outside.

The low speed region was attributed to the tip vortex region. Free stream flow was observed outside of the tip vortex region, and there was a strong shear layer in the edge of the near wake. Turbulence generation would be great in this region because of the high shear rate and large turbulent shear stress. A low velocity region was observed near the shaft approximately one diameter of the blade downstream. This suggests that this region is caused by swirling motion of the near wake. 
Figure 11. Ensemble averaged velocity fields obtained by the PIV measurements. (a) $3.5 \mathrm{~m} / \mathrm{s}$ and $300 \mathrm{rpm}$; (b) $4 \mathrm{~m} / \mathrm{s}$ and $400 \mathrm{rpm}$; (c) $5 \mathrm{~m} / \mathrm{s}$ and $500 \mathrm{rpm}$.

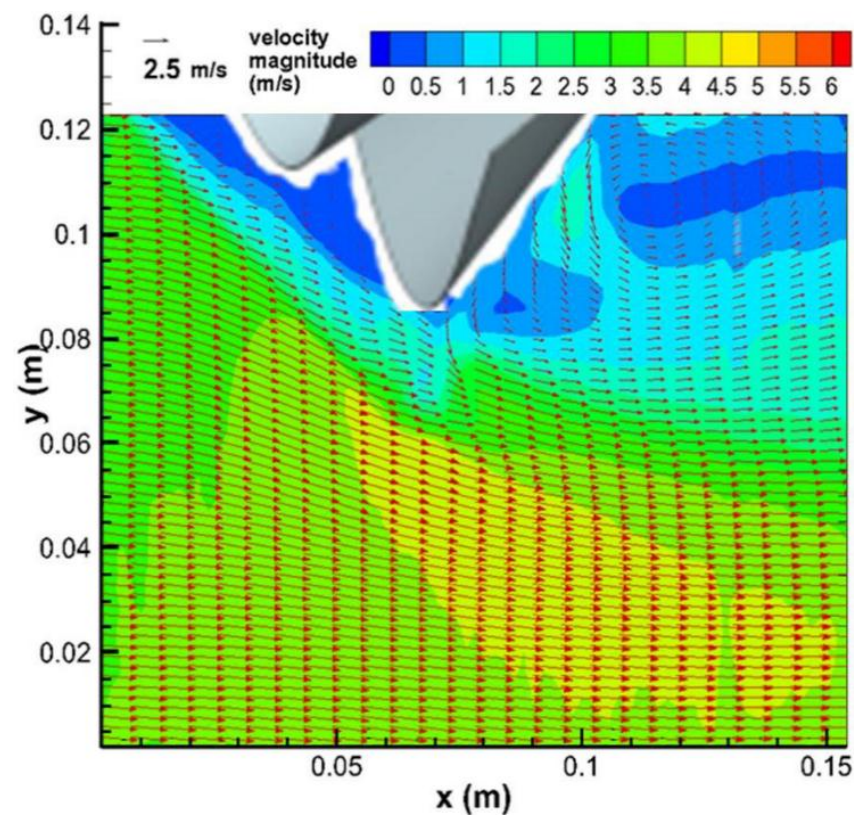

(a)

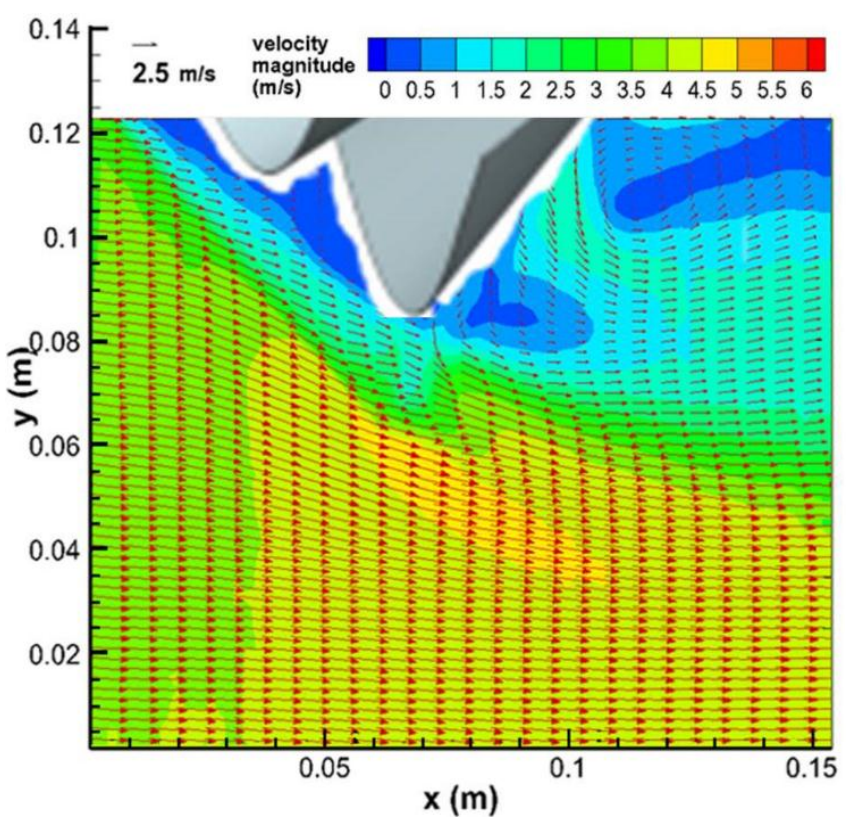

(b)

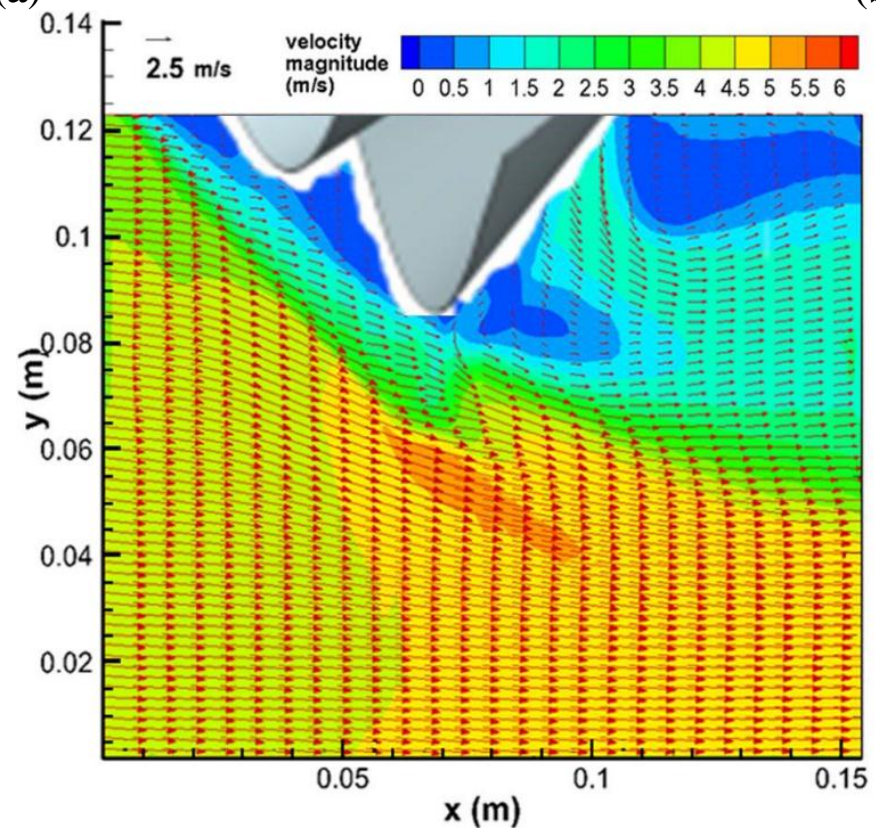

(c)

Figure 12 presents the turbulent kinetic energy (TKE) distribution in the tip vortex of the wind turbine. As the out of plane component (w') of velocity fluctuation was not measured, the TKE was obtained using $1 / 2\left(u^{\prime 2}+2 v^{\prime 2}\right)$ assuming that $w^{\prime}$ is equal to $v^{\prime}$. The turbulent kinetic energy can be generated by random fluctuations and periodical oscillations of unsteady flow. The TKE levels along the tip vortex structures were quite high with the highest TKE level being observed in the wake of the wind turbine model. The high TKE levels in this area are related to the generation of tip vorticity and flow separation around the wind turbine blade, as shown clearly from the ensemble averaged PIV measurement results. 
Figure 12. Turbulent kinetic energy distribution (a) $3.5 \mathrm{~m} / \mathrm{s}$ and $300 \mathrm{rpm}$; (b) $4 \mathrm{~m} / \mathrm{s}$ and $400 \mathrm{rpm}$; (c) $5 \mathrm{~m} / \mathrm{s}$ and $500 \mathrm{rpm}$.

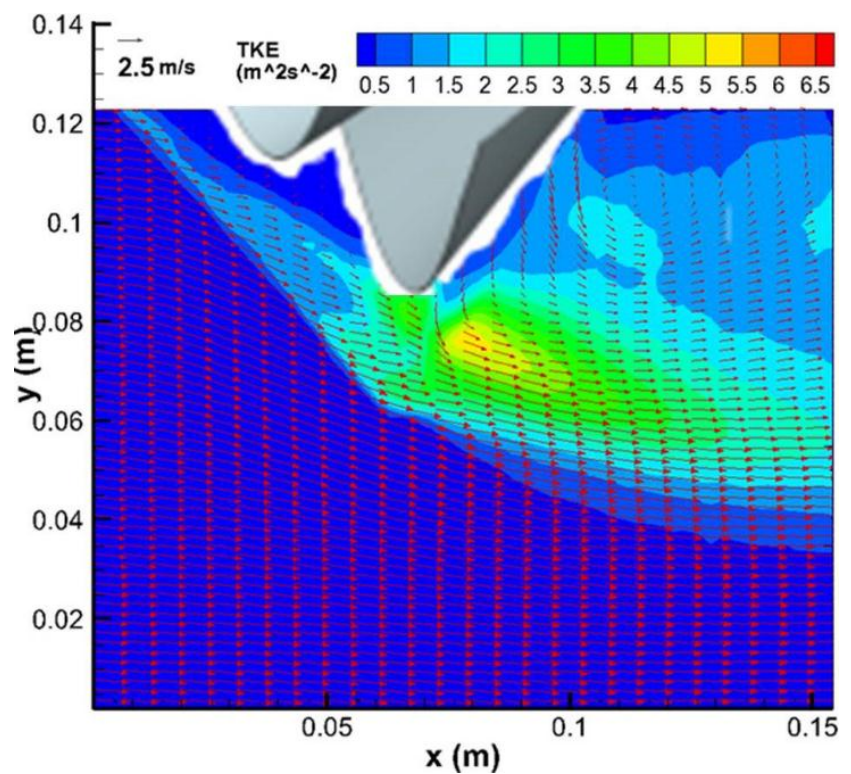

(a)

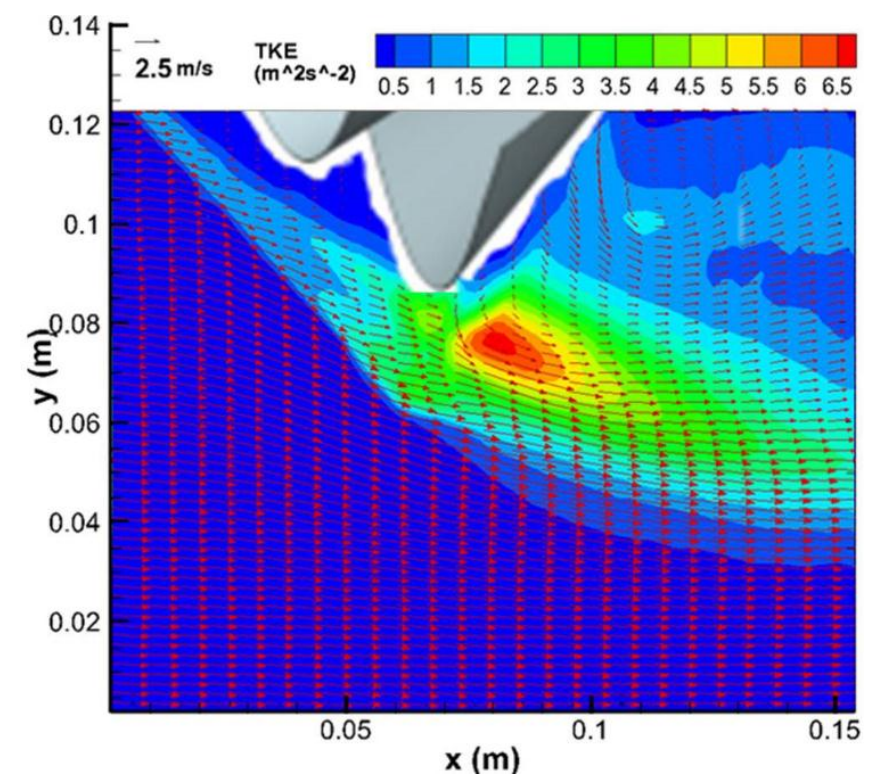

(b)

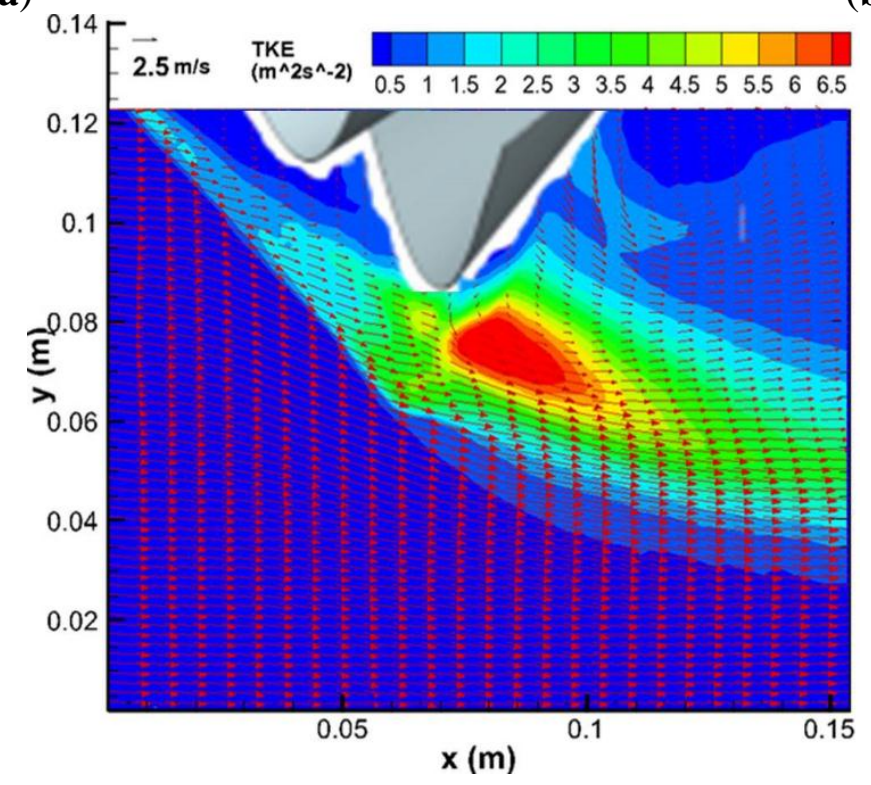

(c)

The aerodynamic characteristics in the near wake of the blades, particularly the tip vortex flow structure, were examined by measuring the instantaneous velocity fields at different phase angles using a PIV experiment. The aerodynamic characteristics at the different relative positions of the Archimedes spiral wind turbine blades can be determined from the contours of the instantaneous velocity and vorticity fields for the different phases during the more comprehensive processing of blade rotation. In general, the aerodynamic characteristics of the instantaneous fields can be reflected more accurately by identifying more phase angles. The range of phase angles is often decided by the number of blades because one cycle of the rotating blades is $360^{\circ}$. Most HAWTs, which include the Archimedes spiral wind turbine, have three centrosymmetrical blades [20]. This means that there are three repeated processes during a single cycle. For one process of the blade of a wind turbine, the range of phase angles 
is $120^{\circ}$. Accordingly, the range of phase angles from $0^{\circ}$ to $120^{\circ}$ was selected because of the three centrosymmetrical blade structure of the Archimedes spiral wind turbine.

A mini-Nd:Yag laser (150 mJ/pulse) was used to obtain a particle image at 15 frames per second. The PIV system was synchronized using the rotational speed meter. To obtain the phase-averaged velocity field, the PIV realizations matching with the phase angles were selected. During the experiment, the blade location and laser firing sequences were recorded. The instantaneous PIV measurement results with an incoming airflow velocity of $3.5 \mathrm{~m} / \mathrm{s}$ and a wind turbine blade rotation speed of $300 \mathrm{rpm}$ were analyzed to determine the instantaneous images of the wake vortex structures at different phase angles, as shown in Figure 13. At the same time, the phase angle was changed from $0^{\circ}$ to $120^{\circ}$ at $20^{\circ}$ intervals. It is difficult to resolve the evolution of tip vortex structure by the standard two-dimensional PIV realizations, however the signature of the tip vortex can be recognized clearly near the outermost blade of the Archimedes spiral wind turbine with respect to the change in phase angle as marked red circles in Figure 13. At a phase angle of $\alpha=0^{\circ}$, the outermost wind turbine blade was in the most downward position. When the phase angle was increased, the outermost wind turbine blade rotated out of the vertical PIV measurement plane. The second wind turbine blade would rotate out into the PIV measurement plane at a phase angle of $\alpha=120^{\circ}$. This shows that as the phase angle is increased, the displacements of the tip vortex structures are transmitted downstream. In contrast to the conventional horizontal wind turbine, the velocity at the core area of the tip vortex induced from the spiral wind turbine is higher due to the spiral-shaped blades, which can increase the airflow speed through the spiral-shaped surface. An examination of the inner blade to the outer layer showed that the movement of the tip vortex coincided approximately with the blade gradual direction. The trajectory of the trailing vortex coincides with the strong shear layer region in the mean velocity field. For all cases of different phase angles, there were no interactions between the tip vortices generated from the three blades.

Figure 13. Instantaneous velocity field of the PIV measurements (Tip phase change)

(a) $\alpha=0^{\circ}$; (b) $\alpha=40^{\circ} ;$ (c) $\alpha=80^{\circ} ;$ (d) $\alpha=120^{\circ}$.

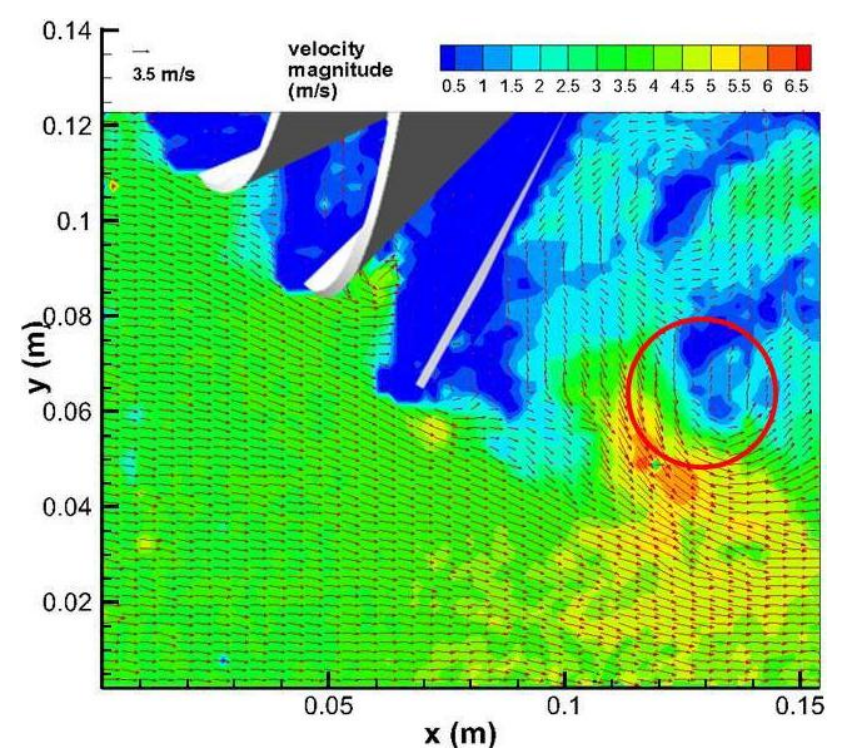

(a)

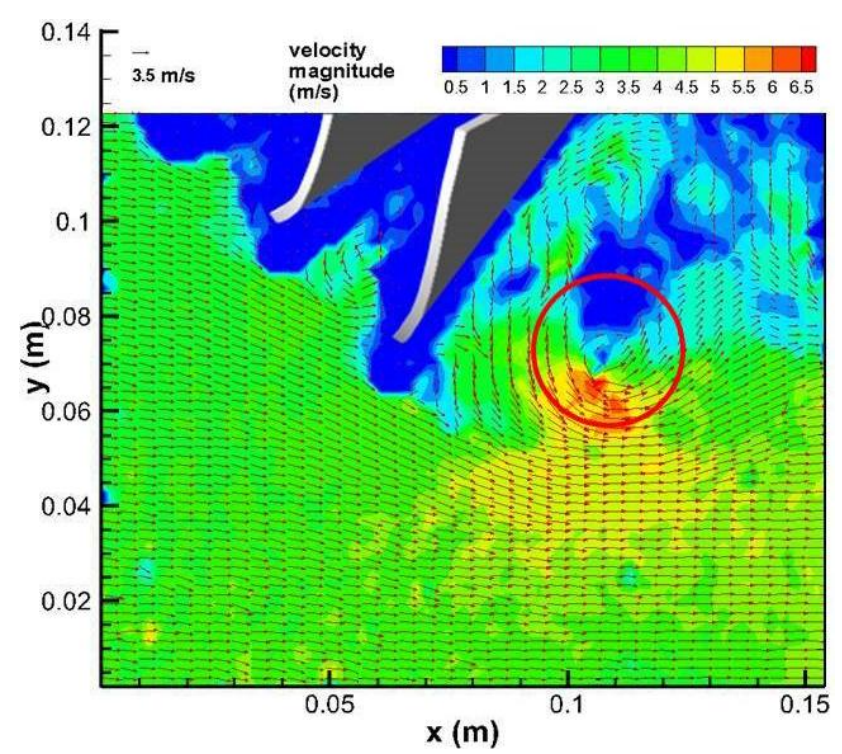

(b) 
Figure 13. Cont.

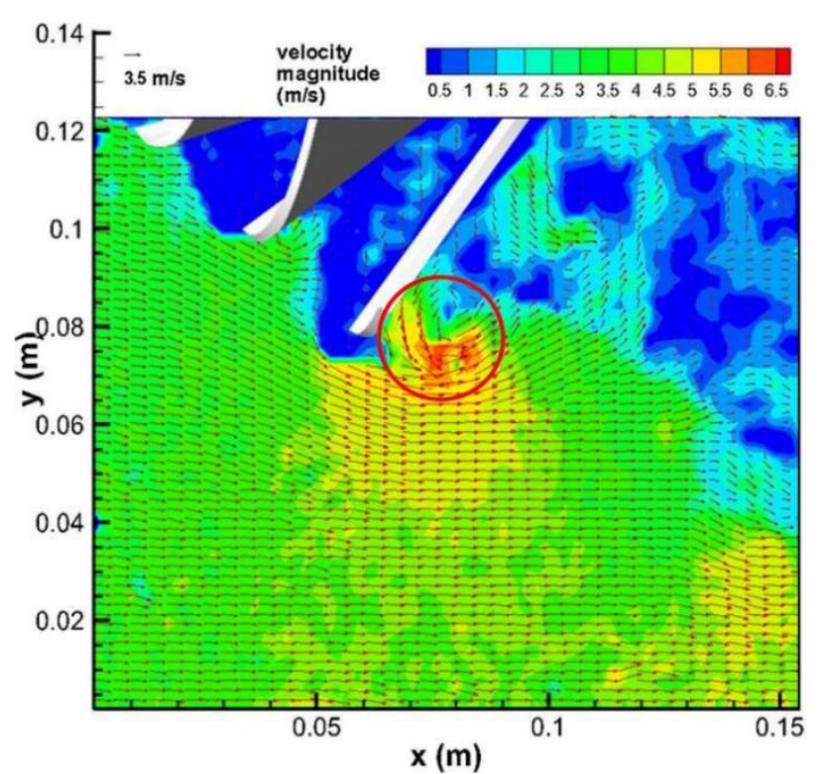

(c)

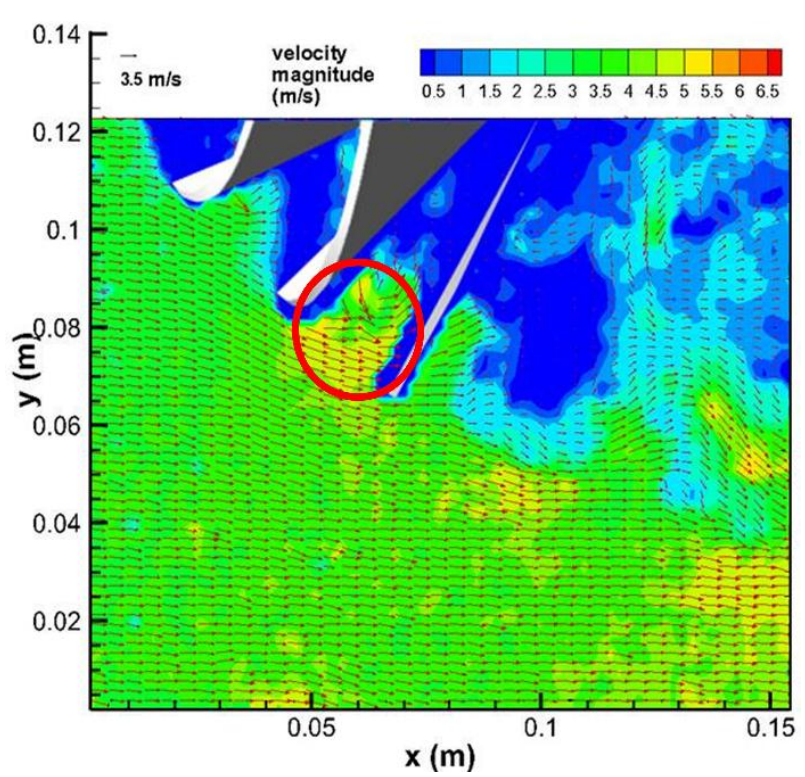

(d)

\subsection{Comparison of the PIV Measurement and CFD Simulations}

Figure 14 presents the PIV and CFD results at $300 \mathrm{rpm}$ and a $3.5 \mathrm{~m} / \mathrm{s}$ wind velocity. To compare the results of the CFD simulation and PIV experiment, the line data of the ensemble-averaged velocity fields were selected as indicated in Figure 14a. The wind velocity of the experiment was higher than that in CFD, and initially, there was a downward tendency that reflected the situation from upstream to downstream through the core of the tip vortex structure. An assessment of those responses on the ensemble averaged velocity field of the PIV measurements showed that as the relative incoming airflow decreases, the tip vortex structures and wake velocity can be influenced more easily because of the spiral-shaped blade. In the lower tip-speed-ratio case, the affected region was wider than that of under higher conditions. The results of the PIV and unsteady CFD showed a similar trend distribution, as shown in Figure 14. The flow was mainly attached and only separated at the core of the tip vortex. This means that the downstream mean velocity in the core of the tip vortex of the PIV is lower than that in the CFD simulation. The boundary sections of the PIV or unsteady state CFD were similar. A comparison of the ensemble-averaged velocity field and the pressure field showed that the flow could not attach on the blade any longer, and the boundary layer separated, resulting in a separated vortex form. This is because the low pressure area of the suction side moved gradually to the blade tip edge when the dynamic pressure was less than the adverse static pressure. Subsequently, the main separated vortex increased further and the region affected was even larger. A comparison of the PIV measurement with the CFD numerical simulation showed that the vector diagram almost agreed with each other, particularly in the shape and vector trend, as well as the position and size of the tip vortex. 
Figure 14. Comparison of the PIV experimental and CFD (steady and unsteady state) results (300 rpm and $U_{\infty}=3.5 \mathrm{~m} / \mathrm{s}$ ). (a) Line Data Extracting Location for the Velocity Profile; (b) Velocity Profile at Line 1; (c) Velocity Profile at Line 2; (d) Velocity Profile at Line 3; (e) Velocity Profile at Line 4.

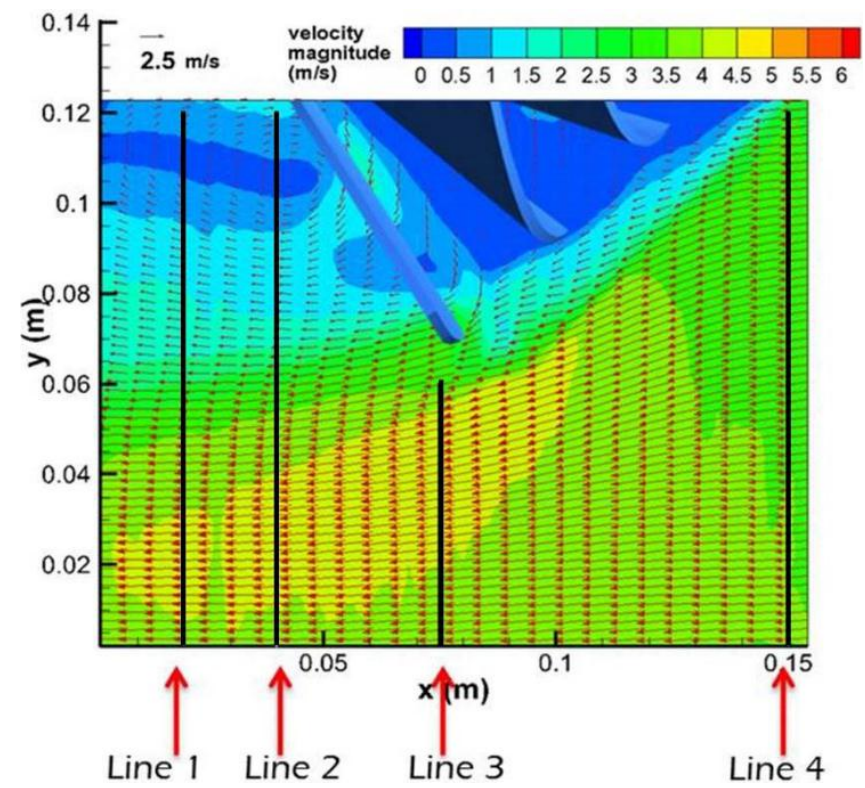

(a)

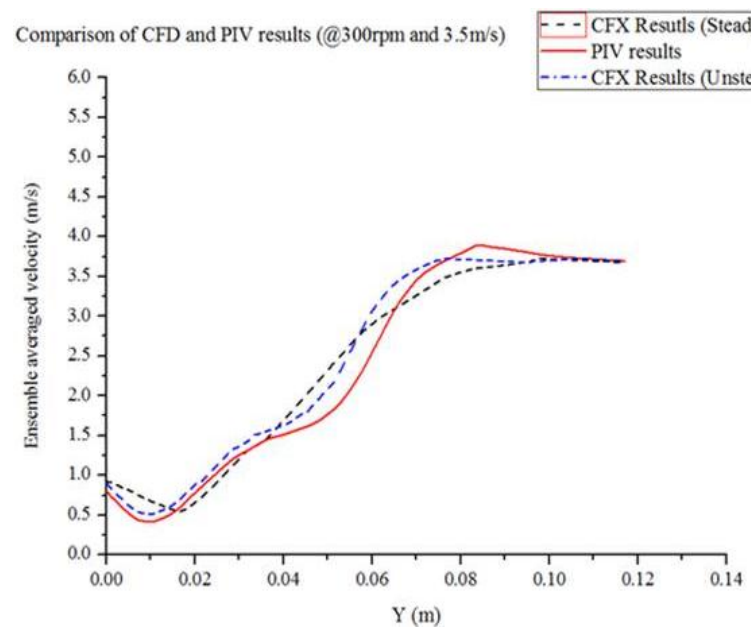

(b)

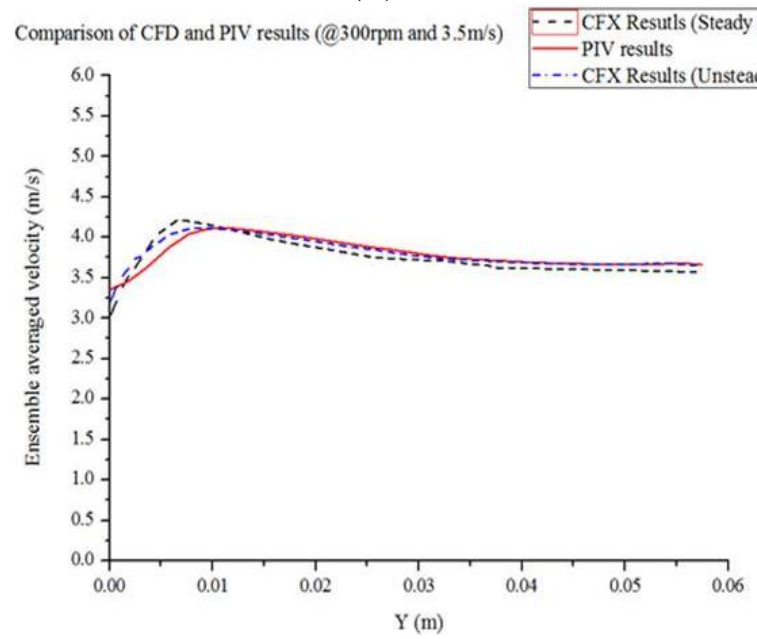

(d)

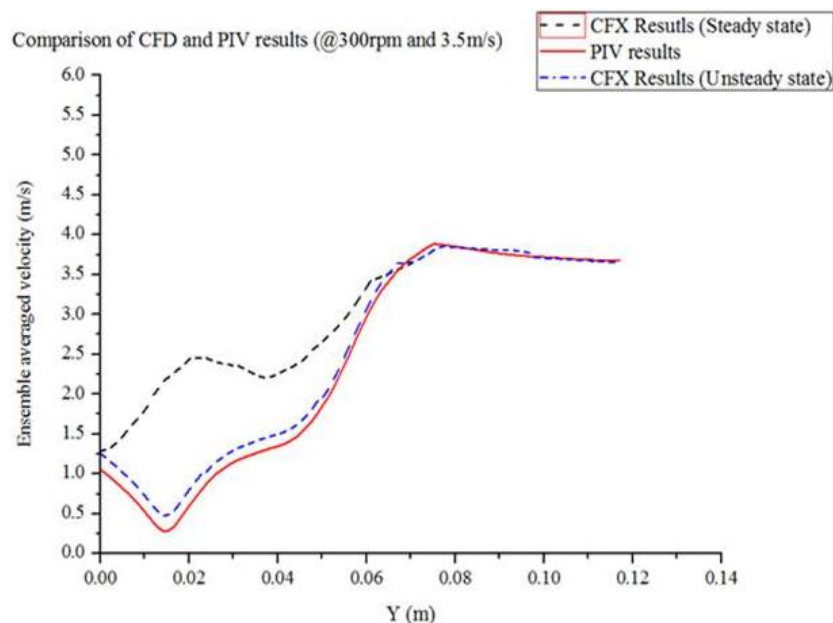

(c)

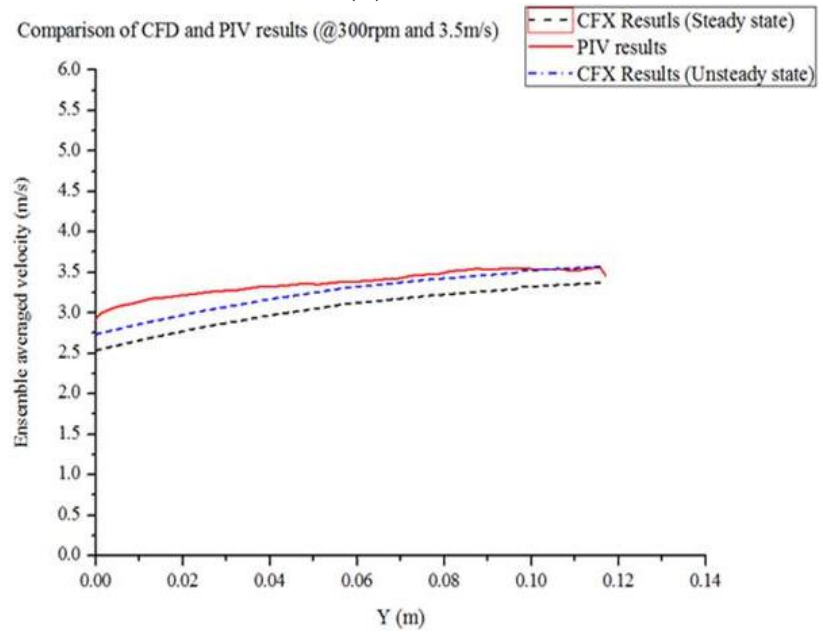

(e) 
In the unsteady state simulation, when the air flow passed the blade, the flow needed to accelerate to pass the blade surface. As the air continuously passed the wind turbine blade, it created a low-pressure area on the tip of the blade because the wind across this area had a higher velocity. On the other hand, the steady state cases were different from the unsteady state because the steady state simulation did not change the relative position between the blade and incoming airflow. In the steady state simulation, the multiple reference frames (MRF) technique was used. The URANS is able to resolve some important structures that occur due to unsteady effects in the rotation, which RANS cannot resolve due to the averaging.

\section{Conclusions}

A new type of HAWT adopting the Archimedes spiral blade format was introduced. A theoretical approach to design the Archimedes wind turbine was addressed. A comparison of the theoretical prediction of the wind turbine performance and that of the full 3D CFD analysis yielded good agreement. The predicted performance characteristics of the $0.5 \mathrm{~kW}$-class Archimedes wind turbine by 3D CFD analysis showed a relatively high power coefficient, $C_{p}=0.25$, compared to the other types of small scale urban usage VAWTs. To validate the CFD method as a design tool for the Archimedes wind turbine and to understand the aerodynamic characteristics in the near wake of the wind turbine, both experiments and numerical simulations were performed. The ensemble averaged and phase averaged velocity fields were obtained using the 2D PIV technique for three different inflow conditions. When there was no friction torque, the blade's rotation speed was affected by the inflow velocity. The maximum pressure differences were observed at the blade tip, suggesting that the most wind energy could be extracted from the outer part of the blade. Spiral shaped tip vortices were observed in both the numerical and experimental results. High TKE values near the blade tips were attributed mainly to the unsteady motion of the tip vortices. A comparison of the velocity distributions in the near wake region of the Archimedes wind turbine between the PIV measurement and numerical simulation was carried out. The unsteady CFD simulation showed better agreement with those of the PIV experiments than the steady state simulation. Based on the overall aerodynamic characteristics, the Archimedes spiral blade was considered to be a suitable horizontal axis type, small scale, urban-usage wind turbine.

\section{Acknowledgments}

This study was supported by New \& Renewable Energy Program (No. 20113030020010, No. 20134030200290) of Korea Institute of Energy Technology Evaluation and Planning (KETEP) and INNOPOLIS Foundation of Korea Ministry of Science, ICT \& Future Planning (No. B2013DD0031).

\section{Author Contributions}

Kyung Chun Kim prepared this manuscript. Ho Seong Ji designed the experimental setup including PIV system. Qian Lu carried out the PIV experiments. Yoon Kee Kim performed the CFD analysis. Joon Ho Baek and Rinus Mieremet fabricated the experimental model. This study was carried out under the supervision of Kyung Chun Kim. All authors carried out data analysis, discussed the results and contributed to writing the paper. 


\section{Nomenclature}

$\gamma: \quad$ Angle between the rotor axis and the tip of the blade;

$\dot{m}_{i n}$ : Mass flow rate into the control volume;

$\dot{m}_{\text {out }}$ : Mass flow rate from the control volume;

$U_{\infty}:$ Free stream velocity;

$W_{\theta}$ : Tangential component for the relative velocity;

$V_{\theta}$ : Tangential component for the absolute velocity;

$V: \quad$ Velocity;

$W: \quad$ Relative Velocity;

$\alpha$ : $\quad$ Angle between $V$ and $V_{\theta}$;

$\beta$ : $\quad$ Angle between $W$ and $W_{\theta}$;

$k$ : Turbulent kinetic energy;

$\omega: \quad$ Specific dissipation rate.

\section{Appendix}

Additional shape parameters can be derived through a geometrical procedure, as shown in Figure A1. Figure Ala presents a schematic diagram of the estimation of the outlet area and relative specification. $L_{1}$ means the distance between the back side center and x position, which is perpendicular to the outer blade tip, $L_{2}$ means the distance between the back side center position and the $\mathrm{x}$ position, which is perpendicular to inner blade tip. From Figure A1a, $A_{\text {out }}$ can be represented by areas $A_{l}$ and $A_{2}$ using the shape parameters, as shown Equations (A1) and (A2):

$$
\begin{gathered}
A_{\text {out }}=A_{1}+A_{2} \\
A_{\text {out }}=\frac{1}{2} R_{1} L_{1}-\frac{1}{2} R_{2} L_{1}+\frac{1}{2}\left(R_{1}-R_{2}\right)\left(L_{2}-L_{1}\right)=\frac{1}{2}\left(R_{1}-R_{2}\right) L_{2}
\end{gathered}
$$

Figure A1b shows the velocity triangle at the outlet boundary, which is the geometrical relation among relative velocity, $W$, absolute velocity, $V$, and tangential velocity, $U$. In this case, $\beta$ is the intermediate angle between $W$ and $U$, and $\dot{m}_{\text {out }}$ is expressed as Equation (A3):

$$
\dot{m}_{\text {out }}=3 \rho A_{\text {out }} W_{\theta}=3 \rho \cdot \frac{1}{2}\left(R_{1}-R_{2}\right) L_{2} \times W \cos \beta=\frac{3}{2} \rho\left(R_{1}-R_{2}\right) L_{2} \times W \cos \beta
$$

where $\dot{m}_{\text {in }}=\dot{m}_{\text {out }}$

$$
\begin{gathered}
\rho U_{\infty} \sin \gamma \times \pi\left(\frac{R_{1}+R_{2}}{2}\right) S_{1}=\frac{3}{2} \rho\left(R_{1}-R_{2}\right) L_{2} \times W \cos \beta \\
\therefore W=\frac{\pi U_{\infty} \sin \gamma\left(R_{1}+R_{2}\right) S_{1}}{3\left(R_{1}-R_{2}\right) L_{2} \times \cos \beta}
\end{gathered}
$$


Figure A1. Definition of $\mathrm{A}_{\text {out }}$ and $U, V$ and $W$.

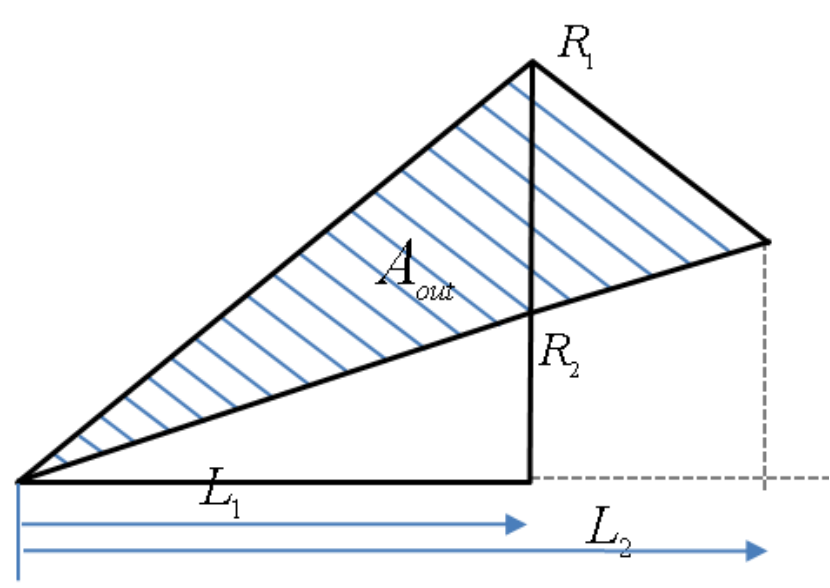

(a)

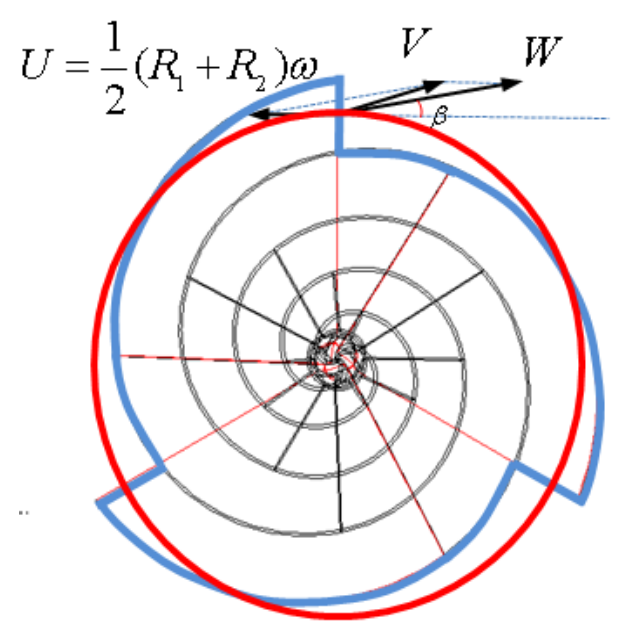

$$
V=U+W
$$

(b)

Figure A2 shows the velocity triangle of $U, V, W$, and $\alpha$ is intermediate angle between $V$ and $V_{\theta}$, $V_{\theta}$ means the perpendicular component of $V$ at the outlet boundary. The absolute velocity, $V$, can be expressed as Equation (A6):

$$
V^{2}=W^{2}+U^{2}-2 W U \cos \beta, V_{\theta}=V \cos \alpha
$$

From Equation (A6), $\alpha$, which is defined by the angle between $V$ and $V_{\theta}$, can be represented by Equation (A7):

$$
\begin{gathered}
V \sin \alpha=W \sin \beta \Rightarrow \sin \alpha=\frac{W}{V} \sin \beta \\
\therefore \alpha=\sin ^{-1}\left(\frac{W}{V} \sin \beta\right)
\end{gathered}
$$

Figure A2. Velocity triangle at the outlet boundary of the blade.

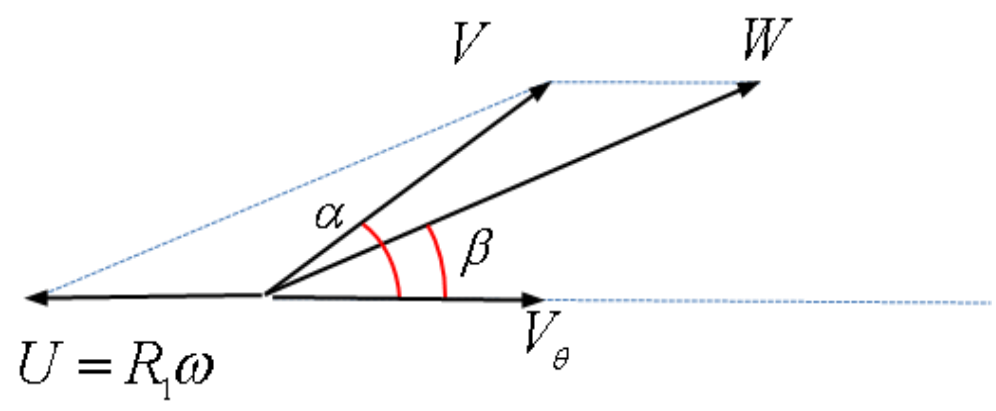

The moment transferred to the fluid by the blade is the same in magnitude as the torque, and has an opposite direction:

$$
T_{\text {shaft }}=\int_{c s}(r \times V) \rho V \cdot \hat{n} d A=\int_{c s}(r V \cos \alpha) \rho(V \cos \alpha) d A=3 \rho V^{2} \cos ^{2} \alpha \int_{0}^{L} r d A
$$

where $\rho$ is the air density, $r$ is the perpendicular distance from the $x$ axis and can be expressed as a function of $x$. As V, $\alpha$ in Equations (A6)-(A9) are known, the torque can be predicted by integrating the 
terms with $r$. Figure A3 presents the outlet boundary, as shown in Figure 3. Equation (A10) can be obtained by rearranging the terms:

$$
\begin{aligned}
\int_{0}^{L} r d A & =\int_{0}^{L_{1}} r d A+\int_{L_{1}}^{L_{2}} r d A \\
& =\frac{\left(R_{2}-R_{1}\right)}{6 L_{1}\left(L_{2}-L_{1}\right)} \times\left[R_{2}\left(2 L_{2}^{3}+L_{1}^{3}+L_{1} L_{2}^{3}\right)-L_{1} R_{1}\left(L_{1}+L_{2}\right)^{2}\right]
\end{aligned}
$$

Figure A3. Finite elements for the area integral.

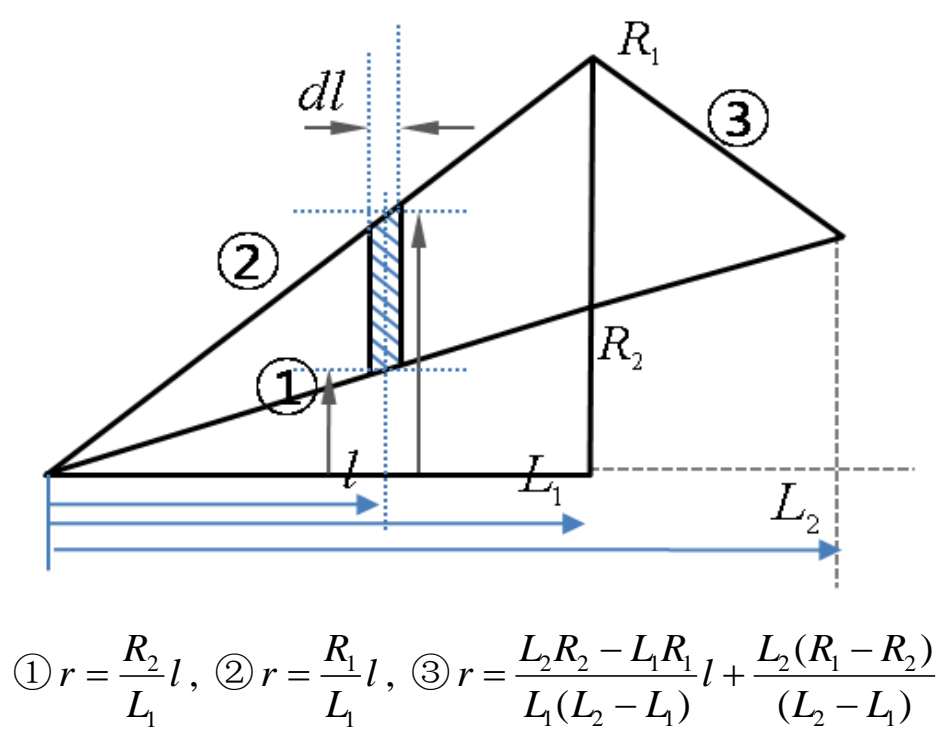

\section{Conflicts of Interest}

The authors declare no conflict of interest.

\section{References}

1. Small Wind World Report Summary 2012; World Wind Energy Association: Bonn, Germany, 2012.

2. Renewables 2011 Global Status Report; Renewable Energy Policy Network for the 21st Century (REN 21): Paris, France, July 2011.

3. Bahaj, A.S.; Myers, L.; James, P.A.B. Urban energy generation: Influence of micro-wind turbine output on electricity consumption in buildings. Energy Build. 2007, 39, 154-165.

4. Ahmed, N.A. A novel small scale efficient wind turbine for power generation. Renew. Energy 2013, 57, 79-85.

5. Simic, Z.; Havelka, J.G.; Vrhovcak, M.B. Small wind turbines-A unique segment of the wind power market. Renew. Energy 2013, 50, 1027-1036.

6. Bortolini, M.; Gamberi, M.; Graziani, A.; Manzini, R.; Pilati, F. Performance and viability analysis of small wind turbines in the European Union. Renew. Energy 2014, 62, 629-639.

7. Arifujjaman, M.; Iqbal, M.T.; Quaicoe, J.E. Energy capture by a small wind-energy conversion system. Appl. Energy 2008, 85, 41-51.

8. Howell, R.; Qin, N.; Edwards, J.; Durrani, N. Wind tunnel and numerical study of a small vertical axis wind turbine. Renew. Energy 2010, 35, 412-422. 
9. Arens, E.A.; Williams, P.B. The effect of wind on energy consumption in buildings. Energy Build. 1977, 1, 77-84.

10. Balduzzi, F.; Bianchini, A.; Carnevale, E.A.; Ferrari, L.; Magnani, S. Feasibility analysis of a Darrieus vertical-axis wind turbine installation in the rooftop of a building. Appl. Energy 2012, 97, 921-929.

11. Hirahara, H.; Hossain, M.Z.; Kawahashi, M.; Nonomura, Y. Testing basic performance of a very small wind turbine designed for multi-purposes. Renew. Energy 2005, 30, 1279-1297.

12. Chen, C.C.; Kuo, C.H. Effects of pitch angle and blade camber on flow characteristics and performance of small-size Darrieus VAWT. J. Vis. 2012, 16, 65-74.

13. Scheurich, R.; Brown, R.E. Modelling the aerodynamics of vertical-axis wind turbines in unsteady wind conditions. Wind Energy 2013, 16, 91-107.

14. Edwards, J.M.; Danao, L.A.; Howell, R.J. PIV measurements and CFD simulation of the performance and flow physics and of a small-scale vertical axis wind turbine. Wind Energy 2013, doi:10.1002/we.1690.

15. Timmer, W.A.; Toet, S. Verslag van de Metingen aan de Archimedes in de Lage-Snelheids Windtunnel van DNW; TU Delft: Delft, The Netherlands, 2009.

16. Lu, Q.; Li, Q.; Kim, Y.K.; Kim, K.C. A study on design and aerodynamic characteristics of a spiral-type wind turbine blade. J. Korean Soc. Vis. 2012, 10, 27-33.

17. Cao, H. Aerodynamics Analysis of Small Horizontal Axis Wind Turbine Blades by Using 2D and 3D CFD Modeling. Master's Thesis, University of the Central Lancashire, Preston, UK, May 2011.

18. Van Bussel, G.J.W. The development of Turby, a small VAWT for the built environment. Presented at Session Advanced Small Turbine Technology, Wind Energy Section, Global Wind Energy Conference 2004, Chicago, IL, USA, 30 March 2004.

19. Ewald, B.F.R. Wind Tunnel Wall Corrections; AGARD-AG-336; North Atlantic Treaty Organization: Brussels, Belgium, October 1998.

20. Kim, K.C.; Yoon, S.Y.; Kim, S.M.; Chun, H.H.; Lee, I. An orthogonal-plane PIV technique for the investigations of three-dimensional vortical structures in a turbulent boundary layer flow. Exp. Fluids 2006, 40, 876-883.

21. Porté-Agel, F.; Wu, Y.T.; Lu, H.; Conzemius, R.J. Large-eddy simulation of atmospheric boundary layer flow through wind turbines and wind farms. J. Wind Eng. Ind. Aerodyn. 2011, 99, 154-168.

22. Choi, N.J.; Nam, S.H.; Jeong, J.H.; Kim, K.C. Numerical study on the horizontal axis turbines arrangement in a wind farm: Effect of separation distance on the turbine aerodynamic power output. J. Wind Eng. Ind. Aerodyn. 2013, 117, 11-17.

23. Fujisawa, N. Velocity measurement and numerical calculations of flow fields in and around Savonius rotors. J. Wind. Eng. Ind. Aerodyn. 1996, 59, 39-50.

(C) 2014 by the authors; licensee MDPI, Basel, Switzerland. This article is an open access article distributed under the terms and conditions of the Creative Commons Attribution license (http://creativecommons.org/licenses/by/4.0/). 